\title{
ON STRONG LIFTINGS ON PROJECTIVE LIMITS
}

\author{
N. D. MACHERAS \\ Department of Statistics and Insurance Science, University of Piraeus, 80 Karaoli and Dimitriou Street, \\ 18534 Piraeus, Greece \\ e-mail:macheras@unipi.gr \\ K. MUSIAŁ \\ Institute of Mathematics, Wroctaw University, Pl. Grunwaldzki 2/4, 50-384 Wroctaw, Poland \\ e-mail:musial@math.uni.wroc.pl \\ and W. STRAUSS ${ }^{\dagger}$ \\ Universität Stuttgart, Fachbereich Mathematik, Institut für Stochastik und Anwendungen, Abteilung für \\ Finanz- und Versicherungsmathematik, Postfach 8011 40, D-70511 Stuttgart, Germany \\ e-mail:strauss@mathematik.uni-stuttgart.de
}

(Received 1 July, 2002; accepted 23 January, 2003)

\begin{abstract}
The main problem investigated in this paper is the following. Assume that we are given a convergent projective system of topological measure spaces ordered by ordinals. When does there exist a consistent system of liftings (densities, linear liftings) on the projective system converging to a lifting (density, linear lifting) on the limit space. We look mainly for strong or strong completion Baire liftings. We reduce the problem to the question about the existence of strong liftings being inverse images of other strong liftings under measure preserving mappings (Proposition 2.4) and then we adapt a condition applied earlier by A. and C. Ionescu Tulcea [14] to get a strong lifting for an arbitrary measure on a product space (Theorem 2.7). In this way we get some results (see Theorems 2.7, 5.3, 5.7, 6.4 and 6.5) extending the well known achievements of A. and C. Ionescu Tulcea [14] and Fremlin [9].

The application of projective limits allows us to carry over results obtained earlier only for product spaces (see e.g. [23], [18], [19], [20], [21]) to more general classes of topological probability spaces. In particular, we can extend the class of spaces for which there is a positive answer to a problem of J. Kupka [17] concerning the permanence of the strong lifting property under the formation of products (see Theorem 6.5).
\end{abstract}

2000 Mathematics Subject Classification. 28A51, 28C15, 60A10.

1. Preliminaries. Given a probability space $(\Omega, \Sigma, \mu)$ the family of all $\mu$-null subsets of $\Omega$ is denoted by $\Sigma_{0}$. For $A, B \in \Sigma$ we write $A=B$ a.e. ( $\mu$ ) iff $A \triangle B$, the symmetric difference of $A$ and $B$, is a $\mu$-null set. The (Carathéodory) completion of $(\Omega, \Sigma, \mu)$ will be denoted by $(\Omega, \widehat{\Sigma}, \widehat{\mu})$. If $\Xi \subset \Sigma$, then $\widehat{\mu \mid} \Xi$ will be the completion of $\mu \mid \Xi \cdot \mathcal{L}^{\infty}(\mu)$ denotes the family of all bounded real-valued $\mu$-measurable functions on $(\Omega, \Sigma, \mu)$. Equivalent functions are not identified. The space of equivalence classes of functions that are $\mu$-integrable (or bounded) is denoted by $L^{1}(\mu)\left(\operatorname{resp}\right.$. by $L^{\infty}(\mu)$ ). The

$\bar{\dagger}$ The authors were partially supported by KBN Grant 5 P03A 01621 and by NATO Grant PST.CLG.977272. 
$\sigma$-algebra generated by a family $\mathcal{L}$ of sets is denoted by $\sigma(\mathcal{L})$. $\mathbf{N}$ and $\mathbf{R}$ stand for the natural numbers and the real numbers respectively. If $M \subseteq \Omega$, then $M^{c}:=\Omega \backslash M$. We use the notion of (lower) density, linear lifting, lifting in the sense of [14, Chapter III] and for every probability space $(\Omega, \Sigma, \mu)$ we denote by $\vartheta(\mu), \mathcal{G}(\mu)$ and $\Lambda(\mu)$ the system of all (lower) densities, linear liftings, and liftings respectively. If $\psi \in \mathcal{G}(\mu)$ then, following [14] we define density $\psi \in \vartheta(\mu)$ by setting

$$
\underline{\psi}(E):=\left\{\psi\left(\chi_{E}\right)=1\right\} \text { for each } E \in \Sigma,
$$

where $\chi_{E}$ denotes the characteristic function of $E$.

Throughout a quadruple $(\Omega, \mathcal{T}, \Sigma, \mu)$ will be called a topological measure space, if $(\Omega, \mathcal{T})$ is a Haussdorff topological space and $(\Omega, \Sigma, \mu)$ is a measure space such that $\mathcal{T} \subseteq \Sigma . \mathcal{B}(\Omega)$ is the Borel $\sigma$-algebra of $(\Omega, \mathcal{T})$. The Baire $\sigma$-algebra of $(\Omega, \mathcal{T})$, i.e. the $\sigma$-algebra generated by the system of all cozero subsets of $\Omega$, is denoted by $\mathcal{B}_{0}(\Omega)$. Let $(\Omega, \Sigma, \mu)$ be a probability space and $\mathcal{T}$ be a topology on $\Omega$. A family $\mathcal{F} \subseteq \Sigma$ is called a measurable network for $(\Omega, \mathcal{T})$ if for each $G \in \mathcal{T}$ there exists a subfamily $\mathcal{G} \subseteq \mathcal{F}$ such that $G=\bigcup \mathcal{G}$. If $\mathcal{F}$ can be taken to be countable, then we say that $\mathcal{F}$ is a countable network. If $(\Omega, \mathcal{T})$ is a (Hausdorff) topological space we denote by $C_{b}(\Omega)$ the space of all bounded continuous functions on $\Omega$. The measure $\mu$ is called $\tau$-additive, if for every incrasing family $\left\langle G_{i}\right\rangle_{i \in I}$ of open subsets of $\Omega$ we have $\mu\left(\bigcup_{i \in I} G_{i}\right)=\sup _{i \in I} \mu\left(G_{i}\right)$.

A Radon measure space is a complete topological measure space $(\Omega, \mathcal{T}, \hat{\mathcal{B}}(\Omega), \mu)$ such that the measure $\mu$ is locally finite, i.e. for every $\omega \in \Omega$ there exists an open set $G$ containing $\omega$ such that $\mu(G)<+\infty$, and inner regular with respect to the compact sets, i.e. $\mu(E)=\sup \{\mu(K): K$ compact, $K \subseteq E\}$ for all $E \in \Sigma$.

For a topological measure space $(\Omega, \mathcal{T}, \Sigma, \mu)$ a lifting $\rho \in \Lambda(\mu)$ is called strong, if $G \subseteq \rho(G)$ for all $G \in \mathcal{T}$, and $\rho$ is called almost strong, if there exists a $N \in \Sigma_{0}$ such that $G \subseteq \rho(G) \cup N$ for all $G \in \mathcal{T}$. We say that a topological measure space $(\Omega, \mathcal{T}, \Sigma, \mu)$ (or just $\mu$ ) has the almost strong lifting property, ASLP for short, if there exists an almost strong lifting $\rho \in \Lambda(\mu)$, and it has the universal strong lifting property (USLP for short), if each $\rho \in \Lambda(\mu)$ is almost strong. For a list of spaces having the USLP the reader should compare [26]. This list of spaces comprises most spaces appearing in applications. For each $\rho \in \Lambda(\mu)$ there exists exactly one (multiplicative) lifting $\widetilde{\rho}$ (in the sense of [14, Chapter III, Section 1, Definition 2]) on $\mathcal{L}^{\infty}(\mu)$ such that $\widetilde{\rho}\left(\chi_{A}\right)=\chi_{\rho(A)}$ for all $A \in \Sigma$ (see [14]). For simplicity we write $\tilde{\rho}=\rho$. If $(\Omega, \mathcal{T})$ is completely regular, then a $\rho \in \Lambda(\mu)$ is strong if and only if $\rho(f)=f$ for every $f \in C_{b}(\Omega)$. If $\mu$ admits a strong lifting then $\mu$ is $\tau$-additive (see [1], Prop. 3).

$\rho \in \Lambda(\mu)$ is called completion Baire, if $\rho(A) \in \widehat{\mathcal{B}}_{0}(\Omega)$ for each $A \in \Sigma$, and $\rho$ is called strong completion Baire, if it is strong and completion Baire.

A measure $\mu$ on $\mathcal{B}_{o}(\Omega)$ is called completion regular if for every Borel set $B$ there exist $A_{1}, A_{2} \in \mathcal{B}_{o}(\Omega)$ such that $A_{1} \subseteq B \subseteq A_{2}$ and $\mu\left(A_{2} \backslash A_{1}\right)=0$. A measure $\mu$ on $\mathcal{B}(\Omega)$ is called completion regular, if its restriction to $\mathcal{B}_{o}(\Omega)$ is completion regular. If $\mu$ is completion regular, then each lifting is completion Baire.

For a complete probability space $(\Theta, \Xi, \nu)$ and $\varphi \in \vartheta(\nu)$ put

$$
\mathcal{T}_{\varphi}:=\{A \in \Xi: A \subseteq \varphi(A)\}
$$

Then $\mathcal{T}_{\varphi}$ is a topology on $\Theta$ called the density topology associated with $\varphi$ (see [14, Chapter V, Section 1]). The same definition holds true for liftings instead of densities and the corresponding topology is called the lifting topology. 
If $\gamma$ is an ordinal, then we will often identify it with the set $\{\alpha<\gamma\}$ of all ordinals less than $\gamma$.I will always be a nonempty set and if $\left\langle\left(\Omega_{i}, \Sigma_{i}, \mu_{i}\right)\right\rangle_{i \in I}$ is a family of probability spaces then, for each $\emptyset \neq J \subseteq I$ we denote by $\left(\Omega_{J}, \Sigma_{J}, \mu_{J}\right)$ the product measure space $\otimes_{i \in J}\left(\Omega_{i}, \Sigma_{i}, \mu_{i}\right)$. $\widehat{\otimes}_{i \in J}\left(\Omega_{i}, \Sigma_{i}, \mu_{i}\right)$ is the completion of $\otimes_{i \in J}\left(\Omega_{i}, \Sigma_{i}, \mu_{i}\right)$. For $\emptyset \neq J \subseteq K \subseteq I$ we denote by $f_{J K}$ the canonical projection from $\Omega_{K}$ onto $\Omega_{J}$ and for each $\emptyset \neq J \subseteq I$ we write $f_{J}:=f_{J I}$. For $J:=\{i\} \subseteq I$ we write $f_{i}:=f_{\{i\}}$. If for each $i \in I$ a topology $\mathcal{T}_{i}$ is given, then $\mathcal{T}_{J}$ is the product topology on $\Omega_{J}$, whenever $\emptyset \neq J \subseteq I$. The collection of all finite subsets of $I$ is denoted by $\mathcal{F}(I)$ and $\kappa(I)$ is the first ordinal of the cardinality $\operatorname{card}(I)$.

If $I=\{\alpha<\gamma\}$ and $0<\beta \leq \gamma$, then instead of $\left(\prod_{\alpha<\beta} \Omega_{\alpha}, \otimes_{\alpha<\beta} \Sigma_{\alpha}, \otimes_{\alpha<\beta} \mu_{\alpha}\right)$ we write $\left(\Omega_{<\beta}, \Sigma_{<\beta}, \mu_{<\beta}\right)$ and, we denote by $f_{(<\alpha)(<\beta)}$ (resp. $\left.f_{<\alpha}\right)$ the canonical projection from $\Omega_{<\beta}$ onto $\Omega_{<\alpha}$ (resp. from $\Omega_{<\gamma}$ onto $\Omega_{<\alpha}$ ) for all $\alpha<\beta<\gamma$. [I] ${ }^{<\kappa}$ is the collection of all non-empty subsets $J$ of $I$ such that $\operatorname{card}(J)<\kappa$.

If $(\Omega, \Sigma, \mu)$ is a probability space we write $\mu^{I}$ for the product measure on $\Omega^{I}$ and $\Sigma^{I}$ for its domain.

For the product $(\Omega, \Sigma, \mu)=\otimes_{i \in I}\left(\Omega_{i}, \Sigma_{i}, \mu_{i}\right)$ of a family $\left\langle\left(\Omega_{i}, \Sigma_{i}, \mu_{i}\right)\right\rangle_{i \in I}$ of complete probability spaces let be given a density $\varphi$ for $\widehat{\mu}$. We recall that $\varphi$ respects coordinates, if for each non-empty subset $J$ of $I$ the inclusion $\varphi\left(\widehat{\Sigma}_{J} \times \Omega_{J^{c}}\right) \subseteq \widehat{\Sigma}_{J} \times \Omega_{J^{c}}$ holds true (cf. [9]).

2. Inverse images of densities and liftings. Let $(\Omega, \Sigma, \mu),(\Theta, \Xi, v)$ be complete probability spaces, $f: \Omega \longrightarrow \Theta$ a measurable function with $\mu\left(f^{-1}(B)\right)=v(B)$ for each $B \in \Xi$, and let $\psi \in \mathcal{G}(\nu)$. A linear lifting $\varphi \in \mathcal{G}(\mu)$ is called an inverse image of $\psi$ under $f$, if

$$
\varphi(g \circ f)=\psi(g) \circ f \text { for each } g \in \mathcal{L}^{\infty}(\nu) .
$$

Let $\tau \in \vartheta(\nu)$. A density $\varphi \in \vartheta(\mu)$ is called an inverse image of $\tau$ under $f$, if

$$
\varphi\left(f^{-1}(E)\right)=f^{-1}(\tau(E)) \text { for each } E \in T .
$$

The definition of an inverse image of a lifting is covered by both definitions.

LeMma 2.1. For complete probability spaces $(\Omega, \Sigma, \mu),(\Theta, \Xi, v)$, densities $\delta \in$ $\vartheta(\mu)$ and $\zeta \in \vartheta(\nu)$, and $a-\Xi$-measurable, measure preserving map $f: \Omega \longrightarrow \Theta$ the following conditions are all equivalent.

(i) The map $f$ is $\mathcal{T}_{\delta}-\mathcal{T}_{\zeta}$-continuous.

(ii) For all $\left.B \in \Xi, f^{-1}(\zeta(B)) \subseteq \delta\left(f^{-1}(B)\right)\right)$.

If in addition $\delta \in \Lambda(\mu)$ and $\zeta \in \Lambda(v)$ then we may add the following equivalent condition.

(iii) The lifting $\delta$ is the inverse image of the lifting $\zeta$.

Proof. Note that for all $B, C \in \Xi$ with $B=C$ a.e.(v) we have $f^{-1}(B)=f^{-1}(C)$ a.e. $(\mu)$ since $v=f(\mu)$.

(i) $\Longrightarrow$ (ii). $B \in \Xi$ implies $\zeta(B) \in \mathcal{T}_{\zeta}$, hence $f^{-1}(\zeta(B)) \in \mathcal{T}_{\delta}$. Consequently $f^{-1}(\zeta(B)) \subseteq \delta\left(f^{-1}(\zeta(B))\right)=\delta\left(f^{-1}(B)\right)$.

(ii) $\Longrightarrow$ (i). $G \in \mathcal{T}_{\zeta}$ means $G \subseteq \zeta(G)$, hence $f^{-1}(G) \subseteq f^{-1}(\zeta(G)) \subseteq \delta\left(f^{-1}(G)\right)$, i.e. $f^{-1}(G) \in \mathcal{T}_{\delta}$.

LEMMA 2.2. Let $(\Omega, \Sigma, \mu),(\Theta, \Xi, v)$ be complete probability spaces and $f: \Omega \longrightarrow$ $\Theta$ a measurable, measure preserving map. Then for each $\psi \in \mathcal{G}(v)$ the collection of inverse images of $\psi$ in non-empty. 
Proof. Let $\mathcal{B}$ be the $\sigma$-subalgebra of $\Sigma$ generated by $f^{-1}(\Xi) \cup \Sigma_{0}$. Define $\varphi \in$ $\mathcal{G}(\mu \mid \mathcal{B})$ by means of $\varphi(g):=\psi(h) \circ f$ for each $g \in \mathcal{L}^{\infty}(\mu \mid \mathcal{B})$ and $h \in \mathcal{L}^{\infty}(v)$ such that $h \circ f=g$ a.e. $(\mu)$. Then, it is enough to extend $\varphi$ in an arbitrary way to $\Sigma$.

The corresponding result for liftings instead of linear liftings can be found in [21, Lemma 2.1].

The following problem however remains open:

QUESTION 2.3. Let $(\Omega, \mathcal{T}, \Sigma, \mu)$ and $(\Theta, \mathcal{S}, \Xi, v)$ be complete topological probability spaces and $f: \Omega \longrightarrow \Theta$ a measure preserving map. Suppose that $v$ admits a strong lifting $\tau$. When does there exist a strong inverse image $\rho \in \Lambda(\mu)$ of $\tau$ under $f$ ?

In general a strong lifting may have empty set of strong inverse images (see Remark 2.5). We know two classes of spaces $(\Omega, \mathcal{T}, \Sigma, \mu)$ admitting strong inverse images of $\tau \in \Lambda(\nu)$ under $f: \Omega \rightarrow \Theta$ if $(\Theta, \mathcal{S}, \Xi, v)$ is fixed. These are the hyperstonian spaces and the extremally disconnected Baire spaces where each set of the first category is closed, endowed with a category measure.

The following proposition solves the problem of the existence of a strong inverse image of a strong lifting under a measure preserving mapping in case of liftings possessing almost strong inverse images.

Proposition 2.4. Let $(\Omega, \mathcal{T}, \Sigma, \mu)$ and $(\Theta, \mathcal{S}, \Xi, v)$ be complete topological probability spaces and $f: \Omega \longrightarrow \Theta$ be a measure preserving map. If $v$ admits a strong lifting $\tau$ which has an almost strong inverse image in $\Lambda(\mu)$, then $\tau$ possesses a strong inverse image $\rho \in \Lambda(\mu)$ under $f$ if and only if the following condition is satisfied:

If $B \in \Xi$ and $G \in \mathcal{T}$ are arbitrary, then

$$
f^{-1}(\tau(B)) \cap G \neq \emptyset \Longrightarrow \mu\left(f^{-1}(B) \cap G\right)>0 .
$$

If $\varphi \in \Lambda(\mu)$ is an almost strong inverse image of $\tau$ and $N \in \Sigma_{0}$ is a set satisfying the inclusion $G \subseteq \varphi(G) \cup N$ for all $G \in \mathcal{T}$, then $\rho$ can be taken to satisfy the equality $\varphi\left|N^{c}=\rho\right| N^{c}$.

Proof. For each $\omega \in N$, let

$$
\begin{gathered}
\mathcal{A}(\omega):=\left\{A: A \in f^{-1}(\Xi), \omega \in \varphi(A)\right\}, \quad \mathcal{E}(\omega):=\{G: G \in \mathcal{T}, \omega \in G\}, \\
\mathcal{H}(\omega):=\{A \cap G: A \in \mathcal{A}(\omega), G \in \mathcal{E}(\omega)\}
\end{gathered}
$$

and $\mathcal{F}(\omega) \subseteq \Sigma$ be the filter defined by

$$
\mathcal{F}(\omega):=\{E \in \Sigma: \exists F \in \mathcal{H}(\omega) \text { with } \quad F \subseteq E \quad \text { a.e. }(\mu)\}
$$

Due to the assumption (1) all members of $\mathcal{H}(\omega)$ and, consequently of $\mathcal{F}(\omega)$ are of positive measure and every $\mathcal{F}(\omega)$ is measure stable.

Define a mapping $\bar{\rho}: \Sigma \rightarrow \Sigma$ by means of

$$
\bar{\rho}(E):=\left[\varphi(E) \cap N^{c}\right] \cup \rho^{*}(E) \text { for each } E \in \Sigma,
$$

where $\rho^{*}(E):=\{\omega \in N: E \in \mathcal{F}(\omega)\}$. It is straightforward to verify that $\bar{\rho} \in \vartheta(\mu)$.

Claim 1. For each $E \in T$ we have $f^{-1}(\tau(E)) \subseteq \bar{\rho}\left(f^{-1}(E)\right)$. 
Proof of claim. Let $E \in T$. If $\omega \in N$ we have

$$
\begin{aligned}
\omega \in f^{-1}(\tau(E)) & \Longleftrightarrow \omega \in \varphi\left(f^{-1}(E)\right) \Longleftrightarrow f^{-1}(E) \in \mathcal{A}(\omega) \subseteq \mathcal{F}(\omega) \\
& \Longrightarrow \omega \in \rho^{*}\left(f^{-1}(E)\right) \Longrightarrow \omega \in \bar{\rho}\left(f^{-1}(E)\right),
\end{aligned}
$$

i.e.

$$
f^{-1}(\tau(E)) \cap N \subseteq \bar{\rho}\left(f^{-1}(E)\right) \cap N \quad \text { for all } \quad E \in T .
$$

If $\omega \notin N$ we have

$$
\omega \in \bar{\rho}\left(f^{-1}(E)\right) \Longleftrightarrow \omega \in \varphi\left(f^{-1}(E)\right) \Longleftrightarrow \omega \in f^{-1}(\tau(E)),
$$

i.e.

$$
\bar{\rho}\left(f^{-1}(E)\right) \cap N^{c}=f^{-1}(\tau(E)) \cap N^{c} \quad \text { for all } \quad E \in T .
$$

From (2) and (3) follows the desired result.

Claim 2. $\bar{\rho}$ is strong.

Proof of claim. Let $G \in \mathcal{T}$ and $\omega \in G$.

If $\omega \in N$ then $G \in \mathcal{E}(\omega) \subseteq \mathcal{F}(\omega)$ hence $\omega \in \rho^{*}(G) \subseteq \bar{\rho}(G)$.

If $\omega \notin N$, then $\omega \in \varphi(G)$ hence $\omega \in \varphi(G) \cap N^{c} \subseteq \bar{\rho}(G)$. Consequently $\bar{\rho}$ is strong. This completes the proof of Claim 2.

By von Neumann [30] there exists a $\rho \in \Lambda(\mu)$ such that

$$
\bar{\rho}(E) \subseteq \rho(E) \text { for each } E \in \Sigma .
$$

Since $\bar{\rho}$ is strong it follows by (4) that $\rho$ is strong.

Claim 3. $\rho$ is an inverse image of $\tau$ under $f$.

Proof of claim. For each $A \in T$ applying (4) and Claim 1 we get

$$
f^{-1}(\tau(A)) \subseteq \bar{\rho}\left(f^{-1}(A)\right) \subseteq \rho\left(f^{-1}(A)\right) .
$$

Consequently, applying the above relation for $A^{c}$ instead of $A$ and the lifting properties we get easily the $\rho$ is an inverse image of $\tau$ under $f$.

Assume now that there exists a strong inverse image $\rho \in \Lambda(\mu)$ of $\tau$ under $f$. Let $B \in \Xi, G \in \mathcal{S}$ and $\omega \in f^{-1}(\tau(B)) \cap G$. We have then

$$
\begin{aligned}
\omega \in f^{-1}(\tau(B)) \cap G & \Leftrightarrow \omega \in \rho\left(f^{-1}(B)\right) \cap G \\
& \Rightarrow \omega \in \rho\left(f^{-1}(B)\right) \cap \rho(G)=\rho\left(f^{-1}(B) \cap G\right),
\end{aligned}
$$

hence $\mu\left(f^{-1}(B) \cap G\right)>0$. Thus, condition (1) holds true. This completes the proof of the proposition.

REMARK 2.5. In general the condition (1) alone does not guarantee the existence of a strong inverse image of a strong lifting. To see it let $(\Omega, \mathcal{T}, \Sigma, \mu)$ be the space from Fremlin's simplification [8] of Losert's example and let $\Theta=\Omega$. Then, let $\Xi$ be generated by $\mu$-null sets, $\mathcal{S}$ be the trivial topology on $\Theta$ and $\nu$ be the restriction of $\mu$ to $\Xi$. Then the relation (1) is obviously satisfied if $f$ is the identity map.

For the proof of the next theorem we need the following lemma, which is a generalization of Proposition 2 from [16]. Its proof is a modification of the proof of Proposition 2 from [16]. 
LEMMA 2.6. Let $\left(\Theta_{1}, \mathcal{S}_{1}\right)$ and $\left(\Theta_{2}, \mathcal{S}_{2}\right)$ be topological spaces such that $\left(\Theta_{1}, \mathcal{S}_{1}\right)$ has a countable network $\mathcal{G}_{1} \subseteq \mathcal{B}\left(\Theta_{1}\right)$. Then $\mathcal{B}_{0}\left(\Theta_{1}\right) \otimes \mathcal{B}_{0}\left(\Theta_{2}\right)=\mathcal{B}_{0}\left(\Theta_{1} \times \Theta_{2}\right)$.

Proof. Let $f$ be a continuous function on $\Theta_{1} \times \Theta_{2}$ and let $\left\{G_{n}: n \in \mathbf{N}\right\}$ be a countable basis of the natural topology of $\mathbf{R}$. Suppose that $\mathcal{G}_{1}=\left\{G_{1}^{m}: m \in \mathbf{N}\right\}$. Then for each $n$ we have $f^{-1}\left(G_{n}\right) \in \mathcal{S}_{1} \times \mathcal{S}_{2}$ and

$f^{-1}\left(G_{n}\right)=\bigcup_{m \in \mathbf{N}}\left(G_{1 n}^{m} \times U_{2 n}^{m}\right) \quad$ where $\quad U_{2 n}^{m}=\bigcup\left\{V: V \in \mathcal{S}_{2} \quad \& \quad G_{1 n}^{m} \times V \subseteq f^{-1}\left(G_{n}\right)\right\}$ and $\left\{G_{1 n}^{m}: m, n \in \mathbf{N}\right\}$ is a sub-collection of $\left\{G_{n}: n \in \mathbf{N}\right\}$.

Denote by $\mathcal{S}_{2}^{*}$ the topology in $\Theta_{2}$ generated by the sets $U_{2 n}^{m}$ for $m, n \in \mathbf{N}$. For every $m, n \in \mathbf{N}$ put

$$
U_{1 n}^{m}:=\bigcup\left\{U: U \in \mathcal{S}_{1} \quad \text { and } \quad U \times U_{2 n}^{m} \subseteq f^{-1}\left(G_{n}\right)\right\}
$$

Then we get

$$
f^{-1}\left(G_{n}\right)=\bigcup_{m \in \mathbf{N}}\left(U_{1 n}^{m} \times U_{2 n}^{m}\right)
$$

Denote by by $\mathcal{S}_{1}^{*}$ the topology in $\Theta_{1}$ generated by the sets $U_{1 n}^{m}$ for $m, n \in \mathbf{N}$.

If $G$ is an open subset of $\mathbf{R}$, then

$$
f^{-1}(G)=\bigcup_{k \in \mathbf{N}} f^{-1}\left(G_{n_{k}}\right)
$$

for some $n_{1}, n_{2}, \ldots$ and so

$$
f^{-1}(G)=\bigcup_{k \in \mathbf{N}} f^{-1}\left(G_{n_{k}}\right)=\bigcup_{m, k \in \mathbf{N}}\left(U_{1 n_{k}}^{m} \times U_{2 n_{k}}^{m}\right) .
$$

It follows that $f$ is $\mathcal{S}_{1}^{*} \times \mathcal{S}_{2}^{*}$-continuous.

Since the topologies $\mathcal{S}_{1}^{*}$ and $\mathcal{S}_{2}^{*}$ have countable bases and $f$ is $\mathcal{S}_{1}^{*} \times \mathcal{S}_{2}^{*}$-continuous, it follows from Proposition 1 of [16] that $f$ is $\mathcal{B}_{0}\left(\Theta_{1}, \mathcal{S}_{1}^{*}\right) \otimes \mathcal{B}_{0}\left(\Theta_{2}, \mathcal{S}_{2}^{*}\right)$-measurable, where $\mathcal{B}_{0}\left(\Theta_{i}, \mathcal{S}_{i}^{*}\right)$ denotes the Baire $\sigma$-algebra of $\Theta_{i}$ with respect to the weaker than $\mathcal{S}_{i}$ topology $\mathcal{S}_{i}^{*}$ for $i=1,2$.

Consequently, $f$ is $\mathcal{B}_{0}\left(\Theta_{1}\right) \times \mathcal{B}_{0}\left(\Theta_{2}\right)$-measurable, hence $\mathcal{B}_{0}\left(\Theta_{1} \times \Theta_{2}\right) \subseteq \mathcal{B}_{0}\left(\Theta_{1}\right) \otimes$ $\mathcal{B}_{0}\left(\Theta_{2}\right)$. But, because of the continuity of the canonical projections $f_{i}: \Theta_{1} \times \Theta_{2} \longrightarrow \Theta_{i}$ for $i=1,2$ the inclusion $\mathcal{B}_{0}\left(\Theta_{1}\right) \otimes \mathcal{B}_{0}\left(\Theta_{2}\right) \subseteq \mathcal{B}_{0}\left(\Theta_{1} \times \Theta_{2}\right)$ holds true in any case, hence the result follows.

The hypothesis $(I T)$ in the next theorem, which is a consequence of Proposition 2.4, traces back to A. and C. Ionescu Tulcea [14, page 115]. It is implied by independence and is equivalent itself to the relation (1) of Proposition 2.4.

THEOREM 2.7. Let $\left(\Theta_{i}, \Sigma_{i}\right)$ be a measurable space, $\mathcal{T}_{i}$ a topology on $\Theta_{i}$ for $i=1,2$, $\Omega=\Theta_{1} \times \Theta_{2}, \Sigma=\Sigma_{1} \otimes \Sigma_{2}$ and let $\mathcal{T}:=\mathcal{T}_{1} \times \mathcal{T}_{2}$ be the product topology on $\Omega$. If $\mu$ is a probability measure on $\Sigma$ supporting $\Omega$ denote by $\mu_{i}$ the image measure of $\mu$ under the canonical projection $f_{i}$ from $\Omega$ onto $\Theta_{i}$. Assume that $\mathcal{T}_{2} \subseteq \widehat{\Sigma}_{2}$ and $\widehat{\mu}_{1}$ admits a strong 
lifting $\tau$ (notice that this yields $\mathcal{T}_{1} \subseteq \widehat{\Sigma}_{1}$ ). Then the following conditions are equivalent:

(i) If $U \in \mathcal{T}_{2}$ and $A \in \widehat{\Sigma}_{1}$ are arbitrary, then

$$
\widehat{\mu}(A \times U)=0 \Longrightarrow \widehat{\mu}_{1}(A) \widehat{\mu}_{2}(U)=0 .
$$

(ii) For each $G_{i} \in \mathcal{T}_{i}$ for $i=1,2$, and for each $A \in \widehat{\Sigma}_{1}$ we have

$$
f_{1}^{-1}(\tau(A)) \cap\left(G_{1} \times G_{2}\right) \neq \emptyset \Longrightarrow \widehat{\mu}\left(f_{1}^{-1}(A) \cap\left(G_{1} \times G_{2}\right)\right)>0 .
$$

and each of them follows from

(iii) There exists a strong inverse image $\rho \in \Lambda(\widehat{\mu})$ of $\tau$ under $f_{1}$.

If the topological space $\left(\Theta_{2}, \mathcal{T}_{2}\right)$ has a countable network $\mathcal{G}_{2} \subseteq \widehat{\Sigma}_{2}$, then all the above conditions are equivalent.

In particular, if condition (iii) holds true then

(a) $\widehat{\Sigma}_{i}=\widehat{\mathcal{B}}\left(\Theta_{i}\right)$ for $i=1,2$ implies $\widehat{\Sigma}=\widehat{\mathcal{B}}(\Omega)$.

(b) If for each $i=1,2$ we have $\Sigma_{i}=\mathcal{B}_{0}\left(\Theta_{i}\right)$ and $\left(\Theta_{2}, \mathcal{T}_{2}\right)$ has a countable network $\mathcal{G}_{2} \subseteq \mathcal{B}\left(\Theta_{2}\right)$, then $\mu_{i}$ is completion regular, $\Sigma=\mathcal{B}_{0}(\Omega)$ and $\mu$ is completion regular.

Proof. (i) $\Longrightarrow$ (ii) Let $G_{i} \in \mathcal{T}_{i}$ for $i=1,2$ and $A \in \Sigma_{1}$ be arbitrary sets satisfying condition

$$
f_{1}^{-1}(\tau(A)) \cap\left(G_{1} \times G_{2}\right) \neq \emptyset .
$$

Assume, if possible, that $\widehat{\mu}\left(f_{1}^{-1}(A) \cap\left(G_{1} \times G_{2}\right)\right)=0$, i.e. $\widehat{\mu}\left(\left(A \times \Theta_{2}\right) \cap\left(G_{1} \times G_{2}\right)\right)=0$, or equivalently $\left.\widehat{\mu}\left(\left(A \cap G_{1}\right) \times G_{2}\right)\right)=0$. So applying condition $(i)$ we get

$$
\widehat{\mu}_{1}\left(A \cap G_{1}\right) \mu_{2}\left(G_{2}\right)=0 .
$$

Since $G_{2} \neq \emptyset$, we have $\widehat{\mu}_{2}\left(G_{2}\right)>0$ because $\widehat{\mu}_{2}$ supports $\Theta_{2}$, hence $\widehat{\mu}_{1}\left(A \cap G_{1}\right)=0$ implying $\tau(A) \cap \tau\left(G_{1}\right)=\emptyset$. Since $\tau$ is strong, the latter implies $\tau(A) \cap G_{1}=\emptyset$ hence $f_{1}^{-1}(\tau(A)) \cap f_{1}^{-1}\left(G_{1}\right)=\emptyset$, or equivalently $f_{1}^{-1}(\tau(A)) \cap\left(G_{1} \times \Theta_{2}\right)=\emptyset$ hence $f_{1}^{-1}(\tau(A)) \cap$ $\left(G_{1} \times G_{2}\right)=\emptyset$, which contradicts (6).

Consequently, we deduce that $\widehat{\mu}\left(f_{1}^{-1}(A) \cap\left(G_{1} \times G_{2}\right)\right)>0$, i.e. condition (ii) holds true.

(ii) $\Longrightarrow$ (i) Let $A \in \Sigma_{1}$ and $U \in \mathcal{T}_{2}$ be arbitrary sets such that $\widehat{\mu}(A \times U)=0$ but $\widehat{\mu}_{1}(A) \widehat{\mu}_{2}(U)>0$. Then $\tau(A) \times U \neq \emptyset$ hence $f_{1}^{-1}(\tau(A)) \cap f_{2}^{-1}(U) \neq \emptyset$ therefore applying condition (ii) we get $\widehat{\mu}\left(f_{1}^{-1}(A) \cap f_{2}^{-1}(U)\right)>0$ or $\widehat{\mu}(A \times U)>0$, a contradiction.

(iii) $\Longrightarrow$ (ii) If there exists a strong inverse image $\rho \in \Lambda(\widehat{\mu})$ of $\tau$ under $f_{1}$ then for arbitrary $A \in \Sigma_{1}, G_{i} \in \mathcal{T}_{i}$ for $i=1,2$ and $\left(\theta_{1}, \theta_{2}\right) \in f_{1}^{-1}(\tau(A)) \cap\left(G_{1} \times G_{2}\right)$ we have

$$
\begin{aligned}
\left(\theta_{1}, \theta_{2}\right) \in f_{1}^{-1}(\tau(A)) \cap\left(G_{1} \times G_{2}\right) & \Leftrightarrow\left(\theta_{1}, \theta_{2}\right) \in \rho\left(f_{1}^{-1}(A)\right) \cap\left(G_{1} \times G_{2}\right) \\
& \Rightarrow\left(\theta_{1}, \theta_{2}\right) \in \rho\left(f_{1}^{-1}(A)\right) \cap \rho\left(G_{1} \times G_{2}\right) \\
& \Leftrightarrow\left(\theta_{1}, \theta_{2}\right) \in \rho\left(f_{1}^{-1}(A) \cap\left(G_{1} \times G_{2}\right)\right),
\end{aligned}
$$

hence $\mu\left(f_{1}^{-1}(A) \cap\left(G_{1} \times G_{2}\right)\right)>0$. Thus, condition (ii) holds true.

(ii) $\Longrightarrow$ (iii) Assume now that $\left(\Theta_{2}, \mathcal{T}_{2}\right)$ has a countable network $\mathcal{G}_{2} \subseteq \Sigma_{2}$. Then, according to [21, Lemma 2.1] there exists a lifting $\varphi \in \Lambda(\widehat{\mu})$ being an inverse image of $\tau$ under $f_{1}$. Since $\tau$ is strong we have

$$
f_{1}^{-1}(G) \subseteq \varphi\left(f_{1}^{-1}(G)\right) \text { for each } \quad G \in \mathcal{T}_{1} .
$$


Set

$$
N:=\bigcup\left\{f_{2}^{-1}(U) \backslash \varphi\left(f_{2}^{-1}(U)\right): U \in \mathcal{G}_{2}\right\}
$$

Then $N \in \widehat{\Sigma}_{0}$.

Let

$$
\mathcal{G}:=\left\{U_{1} \times U_{2}: U_{1} \in \mathcal{T}_{1}, U_{2} \in \mathcal{G}_{2}\right\} .
$$

It follows that $\mathcal{G} \subseteq \widehat{\Sigma}$. For each $U_{1} \times U_{2} \in \mathcal{G}$ applying (7) and (8) we get

$$
\begin{aligned}
U_{1} \times U_{2} & =f_{1}^{-1}\left(U_{1}\right) \cap f_{2}^{-1}\left(U_{2}\right) \\
& \subseteq \varphi\left(f_{1}^{-1}\left(U_{1}\right)\right) \cap\left[\varphi\left(f_{2}^{-1}\left(U_{2}\right)\right) \cup N\right] \\
& =\left[\varphi\left(f_{1}^{-1}\left(U_{1}\right)\right) \cap \varphi\left(f_{2}^{-1}\left(U_{2}\right)\right)\right] \cup\left[\varphi\left(f_{1}^{-1}\left(U_{1}\right)\right) \cap N\right] \\
& \subseteq \varphi\left(U_{1} \times U_{2}\right) \cup N,
\end{aligned}
$$

i.e.

$$
U_{1} \times U_{2} \subseteq \varphi\left(U_{1} \times U_{2}\right) \cup N \quad \text { for each } \quad U_{1} \times U_{2} \in \mathcal{G} .
$$

Let $U \in \mathcal{T}$. Then $U=\bigcup_{n \in \mathbf{N}}\left(U_{1 n} \times U_{2 n}\right)$ where $U_{2 n} \in \mathcal{G}_{2}$ and $U_{1 n}=\bigcup\left\{U_{1}: U_{1} \in \mathcal{T}_{1}\right.$ and $\left.U_{1} \times U_{2 n} \subseteq U\right\}$ for $n \in \mathbf{N}$.

Consequently, we have $U \in \widehat{\Sigma}$, hence applying (9) we get

$$
U \subseteq \varphi(U) \cup N \quad \text { for each } \quad U \in \mathcal{T}
$$

i.e. the lifting $\varphi$ is an almost strong inverse image of $\tau$ under $f_{1}$. Therefore applying Proposition 2.4 we conclude condition (iii).

In particular, if condition (iii) holds true then:

(a) Since $\widehat{\Sigma}_{i}=\widehat{\mathcal{B}}\left(\Theta_{i}\right)$, the canonical projections $f_{i}$ are $\widehat{\mathcal{B}}(\Omega)-\widehat{\mathcal{B}}\left(\Theta_{i}\right)$-measurable for $i=1,2$ hence $\widehat{\Sigma} \subseteq \widehat{\mathcal{B}}(\Omega)$, which combined with (iii) implies $\widehat{\mathcal{B}}(\Omega)=\widehat{\Sigma}$.

(b) If for each $i=1,2$ we have $\Sigma_{i}=\mathcal{B}_{0}\left(\Theta_{i}\right)$ then it follows by Lemma 2.6 that $\Sigma=\mathcal{B}_{0}(\Omega)$. On the other hand, it follows by the assumptions that $\mathcal{T}_{i} \subseteq \widehat{\mathcal{B}}_{0}\left(\Theta_{i}\right)$ therefore the equality $\widehat{\mathcal{B}}_{0}\left(\Theta_{i}\right)=\widehat{\mathcal{B}}\left(\Theta_{i}\right)$ holds true hence $\mu_{i}$ is completion regular for each $i=1,2$. Consequently, applying $(a)$ we get $\widehat{\Sigma}=\widehat{\mathcal{B}}(\Omega)=\widehat{\mathcal{B}}_{0}(\Omega)$ and so $\mu$ is completion regular. This completes the proof of the theorem.

REMARK 2.8. The above theorem remains true (with the same proof) under the weaker assumption that $\mu$ is strictly localizable, i.e. if there exists a disjoint family $\left\langle\Omega_{i}\right\rangle_{i \in I}$ of measurable sets of finite measure such that $\Omega=\bigcup_{i \in I} \Omega_{i}$ and

$$
\begin{gathered}
\Sigma=\left\{E: E \subseteq \Omega, E \cap \Omega_{i} \in \Sigma \quad \forall i \in I\right\}, \\
\mu(E)=\sum_{i \in I} \mu\left(E \cap \Omega_{i}\right) \quad \text { for each } \quad E \in \Sigma .
\end{gathered}
$$

Radon measures are strictly localizable, since they have a concassage (see e.g. [32]).

Theorem 2.7 improves Theorem 4 from [14, Chapter VIII, Section 2], where $\Theta_{2}$ is assumed to be a metrizable space.

REMARKs 2.9. (a) From Theorem 2.7 it follows that the condition (IT) implies the existence of an inverse strong lifting for $\widehat{\mu}$ if $\left(\Theta_{2}, \mathcal{T}_{2}\right)$ has a countable network. It is not 
in general true that the existence of a (not nesseccarily inverse image) strong lifting implies condition $(I T)$ (see Remark 5.10 (b)).

(b) If $\left\langle\left(X_{i}, \mathcal{S}_{i}, \Sigma_{i}, \mu_{i}\right)\right\rangle_{i \in I}$ is a family of complete topological probability spaces such that each $\mu_{i}$ has the $U S L P$ and supports $X_{i}$, and if $(\Theta, \mathcal{S}, \Xi, v)$ is a complete topological probability space admitting a strong lifting $\tau$, then the product space

$$
(\Omega, \mathcal{T}, \Sigma, \mu)=\left(\Theta \times X_{I}, \mathcal{S} \times \mathcal{S}_{I}, \Xi \widehat{\otimes} \Sigma_{I}, v \widehat{\otimes} \mu_{I}\right)
$$

is a complete topological probability space admitting a strong inverse image $\rho \in \Lambda(\mu)$ of $\tau$ under the canonical projection $f$ from $\Omega$ onto $\Theta$. The above assertion follows from [27, Theorem 3.9].

(c) We don't know whether Theorem 2.7 remains true under the assumption that $\widehat{\mu}_{2}$ has the USLP (instead of the stronger assumption that $\left(\Theta_{2}, \mathcal{T}_{2}\right)$ has a countable network $\left.\mathcal{G}_{2} \subseteq \Sigma_{2}\right)$. Notice that the assumption of Theorem 2.7 for $\left(\Theta_{2}, \mathcal{T}_{2}\right)$ implies that $\widehat{\mu}_{2}$ has the USLP but according to the Remark 5.10 (c) the inverse implication is not in general true.

If $\mu$ is the product of the probability measures $\mu_{1}$ and $\mu_{2}$ then according to [22, Theorem 5.5] there is a positive answer to the above question.

3. Projective limits of liftings. We use the notions of projective systems and projective limits of probability spaces in the sense of [29]. For a projective system $\left\langle\Omega_{\alpha}, \Sigma_{\alpha}, \mu_{\alpha}, f_{\alpha \beta}, I\right\rangle$ of probability spaces a family $\left\langle\delta_{\alpha}\right\rangle_{\alpha \in I}$ of densities $\delta_{\alpha} \in \vartheta\left(\mu_{\alpha}\right)$ is called consistent, if

$$
\delta_{\beta} \circ f_{\alpha \beta}^{-1}=f_{\alpha \beta}^{-1} \circ \delta_{\alpha}
$$

for all $\alpha, \beta \in I$ with $\alpha \leq \beta$.

If $\left(\Omega, \Sigma, \mu,\left\langle f_{\alpha}\right\rangle_{\alpha \in I}\right)$ is the projective limit of the above projective system and $\mu_{p}$ is an extension of $\mu$ to a $\sigma$-algebra $\Sigma_{p}$ containing $\Sigma$, then a density $\delta \in \vartheta\left(\mu_{p}\right)$ is called a projective limit or a p-density of the consistent family $\left\langle\delta_{\alpha}\right\rangle_{\alpha \in I}$ if

$$
\delta \circ f_{\alpha}^{-1}=f_{\alpha}^{-1} \circ \delta_{\alpha}
$$

for all $\alpha \in I$. We write in that case $\delta \in \operatorname{proj}_{\alpha \in I} \lim _{\alpha}$ (notice that projective limits of densities are not uniquely defined). We will say also that $\left\langle\delta_{\alpha}\right\rangle_{\alpha \in I}$ is convergent to $\delta$. The same definitions hold true for liftings. We assume throughout that all the canonical projections $f_{\alpha}$ are surjections. We will use the notation $\mathbf{p}$-lifting for a lifting being a projective limit of other liftings.

A family $\left\langle\psi_{\alpha}\right\rangle_{\alpha \in I}$ of linear liftings $\psi_{\alpha} \in \mathcal{G}\left(\mu_{\alpha}\right)$ is called consistent, if for all $\alpha, \beta \in I$ with $\alpha \leq \beta$ we have

$$
\psi_{\beta}\left(g \circ f_{\alpha \beta}\right)=\psi_{\alpha}(g) \circ f_{\alpha \beta} \quad \text { for all } g \in \mathcal{L}^{\infty}\left(\mu_{\alpha}\right) .
$$

A linear lifting $\psi \in \mathcal{G}(\mu)$ is called a projective limit or a linear p-lifting of a consistent family $\left\langle\psi_{\alpha}\right\rangle_{\alpha \in I}$, where $\psi_{\alpha} \in \mathcal{G}\left(\mu_{\alpha}\right)$, if for each $\alpha \in I$ we have

$$
\psi\left(g \circ f_{\alpha}\right)=\psi_{\alpha}(g) \circ f_{\alpha} \text { for all } g \in \mathcal{L}^{\infty}\left(\mu_{\alpha}\right) .
$$

For liftings on complete probability spaces definitions (10) and (12) are equivalent and the same is true for (11) and (13). 
If $I=\kappa$, where $\kappa$ is an infinite ordinal, we say that a projective system $\left\langle\Omega_{\alpha}, \Sigma_{\alpha}, \mu_{\alpha}, f_{\alpha \beta}, \kappa\right\rangle$ of probability spaces is continuous, if for every limit ordinal $\xi<\kappa$ the projective limit of the system $\left\langle\Omega_{\alpha}, \Sigma_{\alpha}, \mu_{\alpha}, f_{\alpha \beta}, \xi\right\rangle$ exists and equals to $\left(\Omega_{\xi}, \Sigma_{\xi}, \mu_{\xi},\left\langle f_{\alpha}\right\rangle_{\alpha<\xi}\right)$. For projective systems of topological probability spaces a similar definition can be formulated, but then the mappings $f_{\alpha \beta}$ are assumed to be in addition continuous.

Let $\left\langle\left(\Omega_{i}, \Sigma_{i}\right)\right\rangle_{i \in I}$ be a family of measurable spaces. If $\mu$ is a measure on $\Sigma_{I}$, then $\left(\Omega_{I}, \Sigma_{I}, \mu\right)$ can be represented as a projective limit of a continuous projective system of probability spaces in the following way.

Fix an index $i_{0} \in I$ and assume that $I=\kappa:=\kappa(I)$ and $i_{0}=0$. Then it is easily seen that $\left\langle\left(\Omega_{<\alpha}, \Sigma_{<\alpha}, \mu_{\alpha}, f_{(<\alpha)(<\beta)}, \kappa\right\rangle\right.$ is a continuous projective system of measure spaces and that $\left(\Omega_{I}, \Sigma_{I}, \mu,\left\langle f_{<\alpha}\right\rangle_{\alpha<\kappa}\right)$ is its projective limit.

Consequently, we may consider the notion of a consistent family $\left\langle\rho_{\alpha}\right\rangle_{\alpha<\kappa}$ of liftings $\rho_{\alpha} \in \Lambda\left(\widehat{\mu}_{\alpha}\right)$ as well as the notion of a projective limit lifting (p-lifting, in short) $\rho$ for the measure $\widehat{\mu}$, i.e. of a lifting $\rho \in \Lambda(\widehat{\mu}) \cap \operatorname{proj}_{\alpha<\kappa} \lim \rho_{\alpha}$.

Proposition 3.1. Let $\kappa$ be an infinite ordinal and $\left\langle\Omega_{\alpha}, \Sigma_{\alpha}, \mu_{\alpha}, f_{\alpha \beta}, \kappa\right\rangle$ a projective system of complete probability spaces. Suppose that $\left(\Omega, \Sigma, \mu,\left\langle f_{\alpha}\right\rangle_{\alpha<\kappa}\right)$ is the projective limit of the above projective system and $\left\langle\psi_{\alpha}\right\rangle_{\alpha<\kappa}$ is a consistent family of linear liftings $\psi_{\alpha} \in \mathcal{G}\left(\mu_{\alpha}\right)$. Then there exists a linear lifting $\psi \in \mathcal{G}(\widehat{\mu})$, which is a projective limit of the system $\left\langle\psi_{\alpha}\right\rangle_{\alpha<\kappa}$. If all $\psi_{\alpha}$ are liftings then $\psi$ can be chosen to be a lifting.

Proof. The only interesting case is when $\kappa$ is a limit ordinal. For every $\alpha<\kappa$ we denote by $\Sigma_{\alpha}^{*}$ the $\sigma$-subalgebra of $\Sigma$ generated by $f_{\alpha}^{-1}\left(\Sigma_{\alpha}\right)$ and by the set of all $\mu$-null subsets of $\Omega$, we set $\mu_{\alpha}^{*}:=\widehat{\mu} \mid \Sigma_{\alpha}^{*}$, and define a linear lifting $\psi_{\alpha}^{*} \in \mathcal{G}\left(\mu_{\alpha}^{*}\right)$ by setting

$$
\psi_{\alpha}^{*}(h):=\psi_{\alpha}(g) \circ f_{\alpha}
$$

for every $h \in \mathcal{L}^{\infty}\left(\mu_{\alpha}^{*}\right)$ with $h=g \circ f_{\alpha} \quad$ a.e. $(\mu)$ for some $g \in \mathcal{L}^{\infty}\left(\mu_{\alpha}\right)$.

For all $\alpha, \beta<\kappa$ with $\alpha \leq \beta$ we have $\Sigma_{\alpha}^{*} \subseteq \Sigma_{\beta}^{*}$ hence $\mathcal{L}^{\infty}\left(\mu_{\alpha}^{*}\right) \subseteq \mathcal{L}^{\infty}\left(\mu_{\beta}^{*}\right)$. A direct calculation shows also that $\psi_{\beta}^{*} \mid \mathcal{L}^{\infty}\left(\mu_{\alpha}^{*}\right)=\psi_{\alpha}^{*}$.

If $\kappa$ is a limit ordinal of countable cofinality, then there is a strictly increasing sequence $\left\langle\alpha_{n}\right\rangle_{n \in \mathbf{N}}$ with supremum $\kappa$. Put for simplicity $\psi_{n}^{*}:=\psi_{\alpha_{n}}$ and $\Sigma_{n}^{*}:=\Sigma_{\alpha_{n}}^{*}$ for all $n \in \mathbf{N}$ and let $\mathcal{U}$ be a free ultrafilter on $\mathbf{N}$. Then $\Sigma_{\kappa}^{*}=\sigma\left(\bigcup_{n \in \mathbf{N}} \Sigma_{n}^{*}\right)$ and we can define $\psi$ by setting

$$
\psi(h):=\lim _{n \in \mathcal{U}} \psi_{n}^{*}\left[E_{\Sigma_{n}^{*}}(h)\right] \quad \text { for } \quad h \in \mathcal{L}^{\infty}(\mu)
$$

where $E_{\Sigma_{n}^{*}}(h)$ denotes the conditional expectation of $h$ with respect to $\Sigma_{n}^{*}$. Using the arguments of the proof of Theorem 2 in [14, Chapter IV, Section 1], we get $\psi \in \mathcal{G}\left(\mu_{\kappa}\right)$. For each $\alpha<\kappa$ there exists an $n \in \mathbf{N}$ such that $\alpha<\alpha_{n}, \psi_{\alpha_{n}}^{*}$ extends $\psi_{\alpha}^{*}$ and $\psi$ extends $\psi_{\alpha}^{*}$. Thus $\psi$ is a projective limit of $\left\langle\psi_{\alpha}\right\rangle_{\alpha<\kappa}$.

If $\kappa$ is a limit ordinal of uncountable cofinality, then we proceed in the standard way. If all $\psi_{\alpha}$ are liftings then define for each $\alpha<\kappa$ a lifting $\widetilde{\psi}_{\alpha} \in \Lambda\left(\mu_{\alpha}^{*}\right)$ by setting

$$
\widetilde{\psi}_{\alpha}(A):=f_{\alpha}^{-1}\left(\psi_{\alpha}(B)\right)
$$

for every $A \in \Sigma_{\alpha}^{*}$ with $A=f_{\alpha}^{-1}(B)$ a.e. $(\widehat{\mu})$ for some $A \in \Sigma_{\alpha}$. It can be easily seen that for any $\alpha \leq \beta<\kappa$ the lifting $\widetilde{\psi}_{\beta}$ extends $\widetilde{\psi}_{\alpha}$. 
Assume now that all $\psi_{\alpha}$ are liftings. If $\kappa$ is of countable cofinality, then there exists a density $\varphi \in \vartheta(\widehat{\mu})$ such that $\varphi$ is an inverse image of $\psi_{\alpha}$ under $f_{\alpha}$ for each $\alpha<\kappa$ (see [10, Lemma 1]). By von Neumann [30] there exists a lifting $\psi \in \Lambda(\widehat{\mu})$ such that $\varphi(E) \subseteq \psi(E)$ for each $E \in \widehat{\Sigma}$. It then follows that

$$
\psi\left(f_{\alpha}^{-1}(A)\right)=f^{-1}\left(\psi_{\alpha}(A)\right) \text { for each } A \in \Sigma_{\alpha}
$$

Consequently, $\psi$ is a projective limit of $\left\langle\psi_{\alpha}\right\rangle_{\alpha<\kappa}$.

If $\kappa$ is of uncountable cofinality, then we define $\psi \in \Lambda(\widehat{\mu})$ by

$$
\psi(E):=\widetilde{\psi}_{\alpha}(E) \text { for each } E \in \Sigma_{\alpha}^{*} \text { for some } \alpha<\kappa
$$

Again $\psi$ is a projective limit of $\left\langle\psi_{\alpha}\right\rangle_{\alpha<\kappa}$.

It is known that not every convergent projective system $\left\langle\Omega_{\alpha}, \Sigma_{\alpha}, \mu_{\alpha}, f_{\alpha \beta}, I\right\rangle$ of complete probability spaces admits a consistent family $\left\langle\rho_{\alpha}\right\rangle_{\alpha \in I}$ of (linear) liftings $\rho_{\alpha}$ (see [27, Example 3.5]). In the next theorem we present a condition guaranteeing the existence of such a consistent family.

THEOREM 3.2. Let $\kappa$ be an infinite ordinal and $\left\langle\Omega_{\alpha}, \Sigma_{\alpha}, \mu_{\alpha}, f_{\alpha \beta}, \kappa\right\rangle$ a continuous projective system of complete probability spaces. Suppose that $\left(\Omega, \Sigma, \mu,\left\langle f_{\alpha}\right\rangle_{\alpha<\kappa}\right)$ is the projective limit of the above system. Then:

(1) There exist a consistent family $\left\langle\psi_{\alpha}\right\rangle_{\alpha<\kappa}$ of linear liftings $\psi_{\alpha} \in \mathcal{G}\left(\mu_{\alpha}\right)$ and a linear lifting $\psi \in \mathcal{G}(\widehat{\mu})$ such that $\psi$ is a projective limit of the system $\left\langle\psi_{\alpha}\right\rangle_{\alpha<\kappa}$. If all $\psi_{\alpha}$ are liftings then $\psi$ can be chosen to be a lifting.

(2) There exist a consistent family $\left\langle\varphi_{\alpha}\right\rangle_{\alpha<\kappa}$ of densities $\varphi_{\alpha} \in \vartheta\left(\mu_{\alpha}\right)$ and a density $\varphi \in \vartheta(\widehat{\mu})$ such that $\varphi$ is a projective limit of the system $\left\langle\varphi_{\alpha}\right\rangle_{\alpha<\kappa}$.

Proof. (1) (A) We construct a consistent family $\left\langle\psi_{\alpha}\right\rangle_{\alpha<\kappa}$ of $\psi_{\alpha} \in \mathcal{G}\left(\mu_{\alpha}\right)$ by induction on $\alpha \leq \kappa$. The inductive hypothesis will be that $\psi_{\gamma}$ extends $\psi_{\beta}$ whenever $\beta \leq \gamma \leq \alpha$. To start the induction for $\alpha=0$ we choose an arbitrary $\psi_{0} \in \mathcal{G}\left(\mu_{0}\right)$.

(B) Inductive step to a successor ordinal $\alpha$. If $\alpha=\beta+1$ where $\beta<\kappa$ and $\psi_{\beta} \in$ $\mathcal{G}\left(\mu_{\beta}\right)$ is known we choose by Lemma 2.2 a $\psi_{\alpha} \in \mathcal{G}\left(\mu_{\alpha}\right)$ such that

$$
\psi_{\alpha}\left(g \circ f_{\beta \alpha}\right)=\psi_{\beta}(g) \circ f_{\beta \alpha} \text { for each } g \in \mathcal{L}^{\infty}\left(\mu_{\beta}\right)
$$

Since $\psi_{\beta}\left(g \circ f_{\delta \beta}\right)=\psi_{\delta}(g) \circ f_{\delta \beta}$ for all $\delta \leq \beta$ and all $g \in \mathcal{L}^{\infty}\left(\mu_{\delta}\right)$, we get $\psi_{\alpha}\left(g \circ f_{\delta \alpha}\right)=$ $\psi_{\delta}(g) \circ f_{\delta \alpha}$ from (14), i.e. the family $\left\langle\psi_{\delta}\right\rangle_{\delta \leq \alpha}$ is consistent.

(C) Inductive step to a limit ordinal $\alpha$. In this case we find by Proposition 3.1 a $\psi_{\alpha} \in \mathcal{G}\left(\mu_{\alpha}\right)$ such that $\psi_{\alpha}$ is a projective limit of $\left\langle\psi_{\beta}\right\rangle_{\beta \leq \alpha}$, i.e. the family $\left\langle\psi_{\beta}\right\rangle_{\beta \leq \alpha}$ is consistent.

(D) Thus the induction can be pushed through to obtain a consistent family $\left\langle\psi_{\alpha}\right\rangle_{\alpha \leq \kappa}$ such that $\psi$ is a projective limit of $\left\langle\psi_{\alpha}\right\rangle_{\alpha<\kappa}$.

The corresponding result for liftings instead of linear liftings can be found in [25, Proposition 2.2].

(2) It follows from (1) that there exists a consistent family $\left\langle\psi_{\alpha}\right\rangle_{\alpha<\kappa}$ of linear liftings $\psi_{\alpha} \in \mathcal{G}\left(\mu_{\alpha}\right)$ and a $\psi \in \mathcal{G}(\widehat{\mu})$ such that $\psi$ is a projective limit of $\left\langle\psi_{\alpha}\right\rangle_{\alpha<\kappa}$. For each $\alpha \leq \kappa$ put $\varphi_{\alpha}:=\underline{\psi}_{\alpha}$. Since $\left\langle\psi_{\alpha}\right\rangle_{\alpha \leq \kappa}$ is consistent, for each $\alpha \leq \beta \leq \kappa$ and each $E \in \Sigma_{\alpha}$ we 
have

$$
\begin{aligned}
\varphi_{\beta}\left(f_{\alpha \beta}^{-1}(E)\right) & =\left\{\psi_{\beta}\left(\chi_{f_{\alpha \beta}^{-1}(E)}\right)=1\right\}=\left\{\psi_{\beta}\left(\chi_{E} \circ f_{\alpha \beta}^{-1}\right)=1\right\} \\
& =\left\{\psi_{\alpha}\left(\chi_{E}\right) \circ f_{\alpha \beta}^{-1}=1\right\}=f_{\alpha \beta}^{-1}\left(\left\{\psi_{\alpha}\left(\chi_{E}\right)=1\right\}\right) \\
& =f_{\alpha \beta}^{-1}\left(\varphi_{\alpha}(E)\right),
\end{aligned}
$$

i.e. $\left\langle\varphi_{\alpha}\right\rangle_{\alpha \leq \kappa}$ is consistent, which means that $\left\langle\varphi_{\alpha}\right\rangle_{\alpha<\kappa}$ is consistent and $\varphi:=\varphi_{\kappa}$ is a projective limit of $\left\langle\varphi_{\alpha}\right\rangle_{\alpha<\kappa}$.

The result (2) of the above theorem has been proven for arbitrary (possibly incomplete) probability spaces and for densities in [24, Section 2, Proposition 1] by a different way.

4. Convergence of systems of strong liftings. In case of arbitrary directed sets we have the following result:

LEMMA 4.1. Let be given a projective system $\left\langle\Omega_{\alpha}, \mathcal{T}_{\alpha}, \Sigma_{\alpha}, \mu_{\alpha}, f_{\alpha \beta}, I\right\rangle$ of complete topological probability spaces converging to $(\Omega, \mathcal{T}, \Sigma, \mu)$ and a consistent family $\left\langle\rho_{\alpha}\right\rangle_{\alpha \in I}$ of liftings $\rho_{\alpha} \in \Lambda\left(\mu_{\alpha}\right)$ converging to $\rho \in \Lambda(\mu)$. Then $\rho \in \Lambda(\mu)$ is strong if and only if each $\rho_{\alpha}$ is strong. The same for densities instead of liftings. In both cases we have

$$
\mathcal{T} \subseteq \operatorname{proj}_{\alpha \in I} \lim \mathcal{T}_{\rho_{\alpha}} \subseteq \mathcal{T}_{\rho} \subseteq \mathcal{B}\left(\Omega, \mathcal{T}_{\rho}\right)=\widehat{\Sigma}
$$

If for each $\alpha \in I$ the topological space $\left(\Omega_{\alpha}, \mathcal{T}_{\alpha}\right)$ is completely regular, then the same is true for linear liftings.

Proof. Since $\left\langle\rho_{\alpha}\right\rangle_{\alpha \in I}$ is a consistent family of densities it follows from Lemma 2.1 that for each $\alpha \leq \beta$ the $\operatorname{map} f_{\alpha \beta}$ is $\mathcal{T}_{\rho_{\beta}}-\mathcal{T}_{\rho_{\alpha}}$-continuous and since $\rho$ is a projective limit of $\left\langle\rho_{\alpha}\right\rangle_{\alpha \in I}$ we have again by Lemma 2.1 that for each $\alpha \in I$ the map $f_{\alpha}$ is $\mathcal{T}_{\rho}-\mathcal{T}_{\rho_{\alpha}}$ continuous.

But for each $\alpha \in I$ the density $\rho_{\alpha}$ is strong hence $\mathcal{T}_{\alpha} \subseteq \mathcal{T}_{\rho_{\alpha}}$ and therefore we get

$$
\mathcal{T} \subseteq \operatorname{proj}_{\alpha<\kappa} \lim \mathcal{T}_{\rho_{\alpha}} \subseteq \mathcal{T}_{\rho} \subseteq \mathcal{B}\left(\Omega, \mathcal{T}_{\rho}\right)=\widehat{\Sigma}
$$

Consequently $\rho$ is strong for $(\Omega, \mathcal{T}, \widehat{\Sigma}, \widehat{\mu})$.

Assume now that $\rho$ is strong. Then for each $\alpha \in I$ and for each $U \in \mathcal{T}_{\alpha}$ we have

$$
f_{\alpha}^{-1}(U) \subseteq \rho\left(f_{\alpha}^{-1}(U)\right)=f_{\alpha}^{-1}\left(\rho_{\alpha}(U)\right)
$$

Since all $f_{\alpha}$ are surjections we then get

$$
U \subseteq f_{\alpha}\left(f_{\alpha}^{-1}\left(\rho_{\alpha}(U)\right)\right)=\rho_{\alpha}(U)
$$

that is $\rho_{\alpha}$ is strong.

Suppose that for each $\alpha \in I$ the topological space $\left(\Omega_{\alpha}, \mathcal{T}_{\alpha}\right)$ is completely regular and that $\left\langle\rho_{\alpha}\right\rangle_{\alpha \in I}$ is a consistent family of strong linear liftings. It follows in the same way as in the proof of Theorem 3.2 that $\left\langle\underline{\rho}_{\alpha}\right\rangle_{\alpha \in I}$ is a consistent family of densities $\underline{\rho}_{\alpha} \in \vartheta\left(\mu_{\alpha}\right)$. To show that for each $\alpha \in I$ the density $\rho_{\alpha}$ is strong, let $U \in \mathcal{T}_{\alpha}$. Since each $\rho_{\alpha}$ is strong, it follows in the same way as in [14, page 105], that $\chi_{U} \leq \rho_{\alpha}\left(\chi_{U}\right)$, 
which implies

$$
\underline{\rho}_{\alpha}(U)=\left\{\omega \in \Omega_{\alpha}: \rho_{\alpha}\left(\chi_{U}\right)(\omega)=1\right\} \supseteq\left\{\omega \in \Omega_{\alpha}: \chi_{U}(\omega)=1\right\}=U .
$$

Consequently, $\rho \in \vartheta(\mu)$ is strong hence $\mathcal{T} \subseteq \widehat{\Sigma}$ as above.

To show that $\rho$ is strong, let $U \in \mathcal{T}$. Since $I$ is directed, the family

$$
\mathcal{U}:=\left\{f_{\alpha}^{-1}\left(U_{\alpha}\right): \alpha \in I, U_{\alpha} \in \mathcal{T}_{\alpha}\right\}
$$

is a basis for the topology $\mathcal{T}$ (cf. [5]), it follows that $U=\cup_{\alpha \in J} f_{\alpha}^{-1}\left(U_{\alpha}\right)$ for some $U_{\alpha} \in \mathcal{T}_{\alpha}$ and $J \subseteq I$. So, we get

$$
\begin{aligned}
\rho\left(\chi_{U}\right) & \geq \rho\left(\chi_{f_{\alpha}^{-1}\left(U_{\alpha}\right)}\right)=\rho\left(\chi_{U_{\alpha}} \circ f_{\alpha}\right) \\
& =\rho_{\alpha}\left(\chi_{U_{\alpha}}\right) \circ f_{\alpha} \geq \chi_{U_{\alpha}} \circ f_{\alpha}=\chi_{f_{\alpha}^{-1}\left(U_{\alpha}\right)}
\end{aligned}
$$

for each $\alpha \in J$. Hence $\rho\left(\chi_{U}\right) \geq \chi_{U}$, i.e. $\rho$ is strong.

Proposition 4.2. Let $\kappa$ be an infinite ordinal and $\left\langle\Omega_{\alpha}, \mathcal{T}_{\alpha}, \Sigma_{\alpha}, \mu_{\alpha}, f_{\alpha \beta}, \kappa\right\rangle$ be a continuous projective system of topological probability spaces. Assume that $\left(\Omega, \mathcal{T}, \Sigma, \mu,\left\langle f_{\alpha}\right\rangle_{\alpha<\kappa}\right)$ is the projective limit of the above projective system and $\left\langle\varphi_{\alpha}\right\rangle_{\alpha<\kappa}$ is a consistent family of strong densities $\varphi_{\alpha} \in \vartheta\left(\widehat{\mu}_{\alpha}\right)$. Then there exists a strong density $\varphi \in \vartheta(\widehat{\mu})$ such that the system $\left\langle\varphi_{\alpha}\right\rangle_{\alpha<\kappa}$ is convergent to $\varphi$.

If all densities $\varphi_{\alpha}$ are liftings, then $\varphi$ can be taken to be a strong lifting. Moreover,

(a) if $\widehat{\Sigma}_{\alpha}=\widehat{\mathcal{B}}\left(\Omega_{\alpha}\right)$ for all $\alpha<\kappa$, then $\widehat{\Sigma}=\widehat{\mathcal{B}}(\Omega)$;

(b) if for each $\alpha<\kappa$ we have that $\Omega_{\alpha}$ is compact, $\Sigma_{\alpha}=\mathcal{B}_{0}\left(\Omega_{\alpha}\right)$ and that $\mu_{\alpha}$ is completion regular, then $\Sigma=\mathcal{B}_{0}(\Omega)$ and $\mu$ is completion regular (consequently, $\varphi$ is completion Baire).

If for each $\alpha<\kappa$ the topological space $\left(\Omega_{\alpha}, \mathcal{T}_{\alpha}\right)$ is completely regular and $\varphi_{\alpha}$ is a strong linear lifting, then $\varphi$ can be taken to be a strong linear lifting.

Proof. The proof for densites and lifting can be found in [27, Theorem 3.1].

If for each $\alpha<\kappa$ the space $\left(\Omega_{\alpha}, \mathcal{T}_{\alpha}\right)$ is completely regular, then it follows by Proposition 3.1 that there exists a $\varphi \in \mathcal{G}(\widehat{\mu}) \cap \operatorname{proj}_{\alpha<\kappa} \lim \varphi_{\alpha}$ which is strong according to Lemma 4.1.

In particular,

(a) if $\widehat{\Sigma}_{\alpha}=\widehat{\mathcal{B}}\left(\Omega_{\alpha}\right)$ for each $\alpha<\kappa$, then relation $\mathcal{T} \subseteq \widehat{\Sigma}$ and the obvious one $\widehat{\Sigma} \subseteq \widehat{\mathcal{B}}(\Omega)$ imply that $\widehat{\Sigma}=\widehat{\mathcal{B}}(\Omega)$.

(b) If for each $\alpha<\kappa$ the measure $\mu_{\alpha}$ is completion regular, $\Sigma_{\alpha}=\mathcal{B}_{0}\left(\Omega_{\alpha}\right)$ and $\Omega_{\alpha}$ is compact, then it follows by [3, Theorem 2.3] that $\Sigma=\mathcal{B}_{0}(\Omega)$ hence $\widehat{\Sigma}=\widehat{\mathcal{B}}_{0}(\Omega)$. Consequently, applying $(a)$ we get $\widehat{\Sigma}=\widehat{\mathcal{B}}(\Omega)=\widehat{\mathcal{B}}_{0}(\Omega)$, which means that $\mu$ is completion regular and $\varphi$ is completion Baire.

In Theorem 3.2 and in Proposition 4.2 it is crucial to have a well ordering of the index set (see [27, Example 3.2]). Below is still another example.

EXAMPLE 4.3. Let $\Omega:=[0,1]^{I}$, where $I$ is an index set with the cardinality of the continuum. Gryllakis and Grekas have constructed in [11] a Radon probability measure $\mu$ on $\Omega$, which supports $\Omega$ and is not completion regular. Denote again by $\mu$ the restriction of $\mu$ to $\mathcal{B}_{0}(\Omega)$. Clearly we have that $\left(\Omega, \mathcal{B}_{0}(\Omega), \mu,\left\langle f_{\alpha}\right\rangle_{\alpha \in \mathcal{F}(I)}\right)$ is the projective limit of the system $\left\langle\Omega_{\alpha}, \mathcal{B}_{0}\left(\Omega_{\alpha}\right), \mu_{\alpha}, f_{\alpha \beta}, \mathcal{F}(I)\right\rangle$, where $\Omega_{\alpha}:=[0,1]^{\alpha}$ and $\mu_{\alpha}:=\mu \mid \widehat{\mathcal{B}}_{0}\left(\Omega_{\alpha}\right)$. In particular, we set $\Omega_{1}:=\Omega_{\{i\}}=[0,1]$ and $\mu_{1}:=\mu_{\{i\}}$ for each $i \in I$. 
It follows from [11, Remark 2.9], that for each $\alpha \in \mathcal{F}(I)$ the measure $\widehat{\mu}_{\alpha}$ is equivalent to the Lebesgue measure $\lambda^{\alpha}$.

According to [20, Theorem 2.1], the Lebesgue measure $\lambda$ on $[0,1]$ admits an admissible strong density $\varphi_{1}$, and since $\lambda$ is equivalent to $\widehat{\mu}_{1}$, we get $\varphi_{1} \in A \vartheta\left(\widehat{\mu}_{1}\right)$. For the definition of an admissible (strong) density or linear lifting we refer to [18] (and [20]) or [19], respectively.

Applying now [18, Theorem 2.6] we find a density $\tilde{\varphi}$ for the completion of $\mu_{1}^{I}$ such that $\tilde{\varphi}$ respects coordinates and

$$
\tilde{\varphi}\left(f_{\{i\}}^{-1}(A)\right)=f_{\{i\}}^{-1}\left(\varphi_{1}(A)\right) \text { for each } \quad i \in I \quad \text { and } \quad A \in \widehat{\mathcal{B}}_{0}\left(\Omega_{1}\right)
$$

Since $\widetilde{\varphi}$ respects coordinates, for each $\alpha \in \mathcal{F}(I)$ there exists exactly one $\varphi_{\alpha} \in \vartheta\left(\mu_{\alpha}\right)$ such that

$$
\tilde{\varphi}\left(f_{\alpha}^{-1}(E)\right)=f_{\alpha}^{-1}\left(\varphi_{\alpha}(E)\right) \text { for each } E \in \widehat{\mathcal{B}}_{0}\left(\Omega_{\alpha}\right)
$$

which implies that $\left\langle\varphi_{\alpha}\right\rangle_{\alpha \in \mathcal{F}(I)}$ is a consistent family of densities.

Since $\varphi_{1}$ is strong, it follows by (15) that $\tilde{\varphi}$ is strong. So applying (16) we get that $\varphi_{\alpha}$ is strong.

Suppose that Proposition 4.2 is true for the directed index set $\mathcal{F}(I)$ instead of $\kappa$. Then there exists a strong $\widehat{\varphi} \in \vartheta(\mu)$ such that $\widehat{\varphi}$ is a projective limit of $\left\langle\varphi_{\alpha}\right\rangle_{\alpha \in \mathcal{F}(I)}$, and consequently, $\widehat{\mathcal{B}}_{0}(\Omega)=\widehat{\mathcal{B}}(\Omega)$ should be true, which means that $\mu$ should be completion regular, contradicting to [11].

The following Claim has been proven also in [11] without using strong densities or liftings.

Claim. There is a countable subset $\alpha$ of I such that the completion of the image measure $\mu_{\alpha}$ of $\mu$ under the canonical projection $f_{\alpha}$ from $[0,1]^{I}$ onto $[0,1]^{\alpha}$ is not equivalent to the Lebesgue measure $\lambda_{\alpha}$ on $[0,1]^{\alpha}$.

Proof of claim. Assume that the assertion is false. Then applying the same arguments as above for $I=\omega_{1}$ we would find a consistent family $\left\langle\rho_{\alpha}\right\rangle_{\alpha<\omega_{1}}$ of strong densities $\rho_{\alpha} \in \vartheta\left(\widehat{\mu}_{\alpha}\right)$. Applying Proposition 4.2 we would get then the completion regularity of $\mu$, contradicting its properties.

Taking into account Lemma 4.1 and Proposition 4.2 one can pose the following question (see also [27, Question 3.4]).

QUESTION 4.4. For which projective systems of topological probability spaces with well-ordered index sets do there exist consistent families of strong liftings?

The next result gives a partial answer to Question 4.4.

THEOREM 4.5. Let $\kappa$ be an infinite ordinal, $\left\langle\Omega_{\alpha}, \mathcal{T}_{\alpha}, \Sigma_{\alpha}, \mu_{\alpha}, f_{\alpha \beta}, \kappa\right\rangle$ be a continuous projective system of topological probability spaces and $\left(\Omega, \mathcal{T}, \Sigma, \mu,\left\langle f_{\alpha}\right\rangle_{\alpha<\kappa}\right)$ be its projective limit. Assume that for all $\alpha$ with $1 \leq \alpha<\kappa$ the measure $\widehat{\mu}_{\alpha}$ supports $\Omega_{\alpha}$, $\left(\Omega_{\alpha}, \mathcal{T}_{\alpha}, \Sigma_{\alpha}, \mu_{\alpha}\right)$ has USLP, and $\widehat{\mu}_{0}$ has a strong lifting $\rho_{0}$. If for each successor ordinal $\delta=\alpha+1<\kappa(\alpha \geq 0)$ and each strong lifting $\rho_{\alpha} \in \Lambda\left(\widehat{\mu}_{\alpha}\right)$ we have

$$
f_{\alpha \delta}^{-1}\left(\rho_{\alpha}(B)\right) \cap G \neq \emptyset \Longrightarrow \mu_{\delta}\left(f_{\alpha \delta}^{-1}(B) \cap G\right)>0
$$


when $B \in \Sigma_{\alpha}^{+}$and $G \in \mathcal{T}_{\delta}$ are arbitrary, then the system admits a consistent family of strong liftings $\rho_{\alpha} \in \Lambda\left(\widehat{\mu}_{\alpha}\right), \alpha \geq 0$, converging to a strong $\rho \in \Lambda(\widehat{\mu}) \cap \operatorname{proj}_{\alpha<\kappa} \lim _{\alpha}$ which is strong w.r.t. $\mathcal{T}^{*}=\operatorname{proj}_{\alpha<\kappa} \lim \mathcal{T}_{\rho_{\alpha}}$.

Proof. We construct by induction on $\delta<\kappa$ a consistent family $\left\langle\rho_{\delta}\right\rangle_{\delta<\kappa}$ of strong liftings $\rho_{\delta} \in \Lambda\left(\widehat{\mu}_{\delta}\right)$. We start the induction with $\rho_{0} \in \Lambda\left(\widehat{\mu}_{0}\right)$.

(a) Inductive step to a successor ordinal $\delta=\alpha+1$. Assume that $\rho_{\alpha} \in \Lambda\left(\widehat{\mu}_{\alpha}\right)$ has been defined. It then follows by Proposition 2.4 that there exists a strong lifting $\rho_{\delta} \in$ $\Lambda\left(\widehat{\mu}_{\delta}\right)$ being an inverse image of $\rho_{\alpha}$ under $f_{\alpha \delta}$.

(b) Inductive step to a limit ordinal $\delta$. It follows from Proposition 4.2 that there exists a strong p-lifting $\rho_{\delta} \in \Lambda\left(\widehat{\mu}_{\delta}\right) \cap \operatorname{proj}_{\alpha<\delta} \lim \rho_{\alpha}$. When $\delta=\kappa$, we get the result.

(c) Since $\left\langle\rho_{\alpha}\right\rangle_{\alpha<\kappa}$ is a consistent family of strong liftings, it follows by Proposition 4.2 that there exists a strong lifting $\rho \in \Lambda(\widehat{\mu})$ being a projective limit of $\left\langle\rho_{\alpha}\right\rangle_{\alpha<\kappa}$.

(d) It follows from Lemma 2.1 that for each $\alpha \leq \beta<\kappa$ the map $f_{\alpha \beta}$ is $\mathcal{T}_{\rho_{\beta}}-\mathcal{T}_{\rho_{\alpha}}$-continuous hence $\left\langle\Omega_{\alpha}, \mathcal{T}_{\rho_{\alpha}}, \widehat{\Sigma}_{\alpha}, \widehat{\mu}_{\alpha}, f_{\alpha \beta}, \kappa\right\rangle$ is a projective system of complete topological probability spaces. So, applying Lemma 4.1 we get that $\rho$ is strong with respect to $\mathcal{T}^{*}$ and that $\mathcal{T} \subseteq \mathcal{T}^{*} \subseteq \mathcal{T}_{\rho} \subseteq \widehat{\Sigma}$.

Question 4.6. Assume that $\left\langle\Omega_{\alpha}, \mathcal{T}_{\alpha}, \Sigma_{\alpha}, \mu_{\alpha}, f_{\alpha \beta}, \kappa\right\rangle$ is a continuous projective system of arbitrary not necessarily complete topological probability spaces such that $\mu_{0}$ has a strong density and for each $1 \leq \alpha<\kappa$ the measure $\mu_{\alpha}$ supports $\Omega_{\alpha}$ and has the $U S D P$, i.e. each density for $\mu_{\alpha}$ is almost strong. Does there exist a consistent family $\left\langle\psi_{\alpha}\right\rangle_{\alpha<\kappa}$ of strong densities $\psi_{\alpha} \in \vartheta\left(\mu_{\alpha}\right)$ ?

REMARK 4.7. In Theorem 4.5 we have in general that $\mathcal{T} \neq \mathcal{T}^{*}=\operatorname{proj}_{\alpha<\kappa} \lim \mathcal{T}_{\alpha} \neq \mathcal{T}_{\rho}$. In fact, for each $\delta<\kappa$ let $\mathcal{S}_{<\delta}, f_{<\delta}$ and $\rho_{\delta}$ be as in Theorem 5.2, and let $A \in$ $\mathcal{S}_{\rho_{\delta}} \backslash \mathcal{S}_{<\delta}$. Then $f_{<\delta}^{-1}(A) \in \mathcal{S}^{*}$, where $\mathcal{S}^{*}$ denotes the projective limit of the projective system $\left\langle\mathcal{S}_{\rho_{\delta}}\right\rangle_{\delta<\kappa}$, but $f_{<\delta}^{-1}(A) \notin \mathcal{S}_{I}$, since if we assume that $f_{<\delta}^{-1}(A) \in \mathcal{S}_{I}$ we should have $f_{<\delta}\left(f_{<\delta}^{-1}(A)\right)=A \in \mathcal{S}_{<\delta}$ because $f_{<\delta}$ is surjective and open, a contradiction. Consequently, $\mathcal{T} \neq \mathcal{T}^{*}$.

To show that in general $\mathcal{T}^{*} \neq \mathcal{T}_{\rho}$, consider the continuous projective system $\left\langle D_{n}\right.$, $\left.\mathcal{T}_{n}, \mathcal{P}\left(D_{n}\right), \mu_{n}, f_{m n}, \mathbf{N}\right\rangle$, where $D_{n}:=\{0,1\}^{n}$ endowed with the discrete topology $\mathcal{T}_{n}$, $\mu_{1}(\{0\})=\mu_{1}(\{1\})=1 / 2, \mu_{n}:=\mu_{1}^{n}$ and $f_{m n}$ is the canonical projection from $D_{n}$ onto $D_{m}$ for each $m \leq n \in \mathbf{N}$. Denote by $\left(\Omega, \mathcal{T}, \Sigma, \mu,\left\langle f_{n}\right\rangle_{n \in \mathbf{N}}\right)$ its projective limit. Then $\mu$ is the usual product measure $\mu_{1}^{\mathbf{N}}$ on $\Omega:=\{0,1\}^{\mathbf{N}}$.

For each $n \in \mathbf{N}$ put $\rho_{n}:=i d_{D_{n}} \in \Lambda\left(\mu_{n}\right)$. Then $\left\langle\rho_{n}\right\rangle_{n \in \mathbf{N}}$ is a consistent sequence of strong liftings, and so by Theorem 4.2 there exists a strong lifting $\rho \in \Lambda(\widehat{\mu})$ being a projective limit of $\left\langle\rho_{n}\right\rangle_{n \in \mathbf{N}}$. Clearly for all $n \in \mathbf{N}$ we have $\mathcal{T}_{n}=\mathcal{T}_{\rho_{n}}$ hence $\mathcal{T}^{*}=\mathcal{T}$. Suppose that $\mathcal{T}^{*}=\mathcal{T}_{\rho}$. Then the topological space $(\Omega, \mathcal{T})$ should be extremally disconnected (cf. [14, Chapter V, Section 3]), a contradiction, because $\Omega$ is homeomorphic to the Cantor set $\Delta \subseteq \mathbf{R}$ endowed with the subspace topology (see e.g. [5, Chapter IV, Section 4]) which is not extremaly disconnected.

5. Measures on product spaces. Throughout this section $I$ is an arbitrary index set, $\kappa=\kappa(I),\left\langle\left(X_{i}, \mathcal{S}_{i}\right)\right\rangle_{i \in I}$ and $\left\langle\Xi_{i}\right\rangle_{i \in I}$ are families of topological spaces $\left(X_{i}, \mathcal{S}_{i}\right)$ and $\sigma$-algebras $\Xi_{i}$ on $X_{i}$, respectively, $v$ is a probability measure on $\Xi_{I}$ and $v_{J}$ the image measure of $v$ under $f_{J}$ for each $J \in[I]^{<\kappa}$. To prove the next result, we need first the following lemma. 
LEMMA 5.1. Let $\left\langle\left(X_{i}, \mathcal{S}_{i}\right)\right\rangle_{i \in I}$ be a family of topological spaces and $\mu$ a probability measure on $\mathcal{B}\left(X_{I}\right)$ supporting $X_{I}$ and being $\tau$-additive. Assume that

$$
\mathcal{B}_{0}\left(X_{J}\right)=\otimes_{i \in J} \mathcal{B}_{0}\left(X_{i}\right) \quad \text { for each finite } \quad J \subseteq I .
$$

Then $\mathcal{B}_{0}\left(X_{I}\right)=\otimes_{i \in I} \mathcal{B}_{0}\left(X_{i}\right)$.

Proof. (a) Since $\mu$ has full support the space $X_{I}$ satisfies the $c c c$, i.e. every pairwise disjoint family of open sets in $X_{I}$ is countable, and so every element of $\mathcal{B}_{0}\left(X_{I}\right)$ is determined by countably many coordinates; in fact the elements of $\mathcal{B}_{0}\left(X_{I}\right)$ have the form $f_{J}^{-1}(C)$, where $C \in \mathcal{B}_{0}\left(X_{J}\right)$ and $J$ is countable (see [31]). Thus $I=\mathbf{N}$ may be assumed without loss of generality.

(b) In case $I=\mathbf{N}$, let $f: X_{\mathbf{N}} \longrightarrow \mathbf{R}$ be a continuous function. Fix $y=\left\langle y_{n}\right\rangle_{n \in \mathbf{N}}$ in $X_{\mathbf{N}}$ and define $f_{n}: X_{\mathbf{N}} \longrightarrow \mathbf{R}$ by means of

$$
f_{n}\left(\left\langle x_{n}\right\rangle_{n \in \mathbf{N}}\right):=f\left(\left\langle x_{1}, \ldots, x_{n}, y_{n+1}, y_{n+2}, \ldots\right\rangle\right) \text { for } n \in \mathbf{N} \text {. }
$$

Then each $f_{n}$ is a continuous function and $\lim _{n \rightarrow \infty} f_{n}(x)=f(x)$. Since the function $f_{n}$ depends only on the first $n$ coordinates, without loss of generality the result can be reduced to the case where $I$ is finite. So the result follows.

The condition (18) in the above lemma is satisfied if for each $i \in I$ the space $\left(X_{i}, \mathcal{S}_{i}\right)$ is locally compact (see [3, lemma on page 326]), or it has a countable network consisting of elements of $\mathcal{B}\left(X_{i}\right)$ (see Lemma 2.6), or it is second countable or a Suslin space (see [16]).

The condition $(I T)$ of the next theorem corresponds to $(I T)$ from Theorem 2.7 but now for infinite products.

THEOREM 5.2. Let I be an arbitrary infinite index set and let $\kappa=\kappa(I)$. For each $i \in I$ let $\left(X_{i}, \mathcal{S}_{i}\right)$ be a topological space and let $\Xi_{i}$ be a $\sigma$-algebra on $X_{i}$. If $v$ is a probability measure on $\Xi_{I}$, then for every $J \in[I]^{<\kappa}$ denote by $v_{J}$ the image measure on $\Xi_{J}$ of $\mu$ under the canonical projection $f_{J}$. For every $J \in[I]^{<\kappa}$ assume that $\mathcal{S}_{J} \subseteq \widehat{\Xi}_{J}$ and $\widehat{v}_{J}$ supports $X_{J}$. Fix an index $i_{0} \in I$ and assume that $v_{i_{0}}$ admits a strong lifting and that for every $J \in[I]^{<\chi}\left\{i_{0}\right\}$ the measure $\widehat{v}_{J}$ has the USLP. Then the following conditions are equivalent:

(i) If $J \in[I]^{<\kappa}, i \in I \backslash J, B \in \widehat{\Xi}_{J}$ and $U \in \mathcal{S}_{i}$ then

$$
\widehat{v}_{J \cup\{i\}}(B \times U)=0 \Longrightarrow \widehat{v}_{J}(B) \widehat{v}_{\{i\}}(U)=0 .
$$

(ii) For every $J \in[I]^{<\kappa}, i \in I \backslash J, B \in \widehat{\Xi}_{J}$, for every elementary open set $U \in \mathcal{S}_{J \cup\{i\}}$ and for every strong lifting $\rho_{J} \in \Lambda\left(\widehat{v}_{J}\right)$ we have

$$
f_{J, J \cup\{i\}}^{-1}\left(\rho_{J}(B)\right) \cap U \neq \emptyset \Longrightarrow \widehat{v}_{J \cup\{i\}}\left(f_{J, J \cup\{i\}}^{-1}(B) \cap U\right)>0 .
$$

If one of the above is satisfied, then there exists a strong lifting $\rho \in \Lambda(\widehat{v})$. Moreover,

(a) if $\widehat{\Xi}_{J}=\widehat{\mathcal{B}}\left(X_{J}\right)$ for every $J \in[I]^{<\kappa}$, then $\widehat{\Xi}_{I}=\widehat{\mathcal{B}}\left(X_{I}\right)$;

(b) if $\Xi_{K}=\mathcal{B}_{0}\left(X_{K}\right)$ for every finite $K \subseteq I$ and $v_{J}$ is completion regular for every $J \in[I]^{<\kappa}$, then $\Xi_{J}=\mathcal{B}_{0}\left(X_{J}\right)$ for every $J \subseteq I$ and $v$ is completion regular (hence $\rho$ is completion Baire).

The whole thesis remains true when card $(I)=\omega_{1}$ and all spaces $\left(X_{i}, \mathcal{T}\right)_{i \neq i_{0}}$ satisfy the second axiom of countability. 
Proof. The equivalence of ( $i$ ) and (ii) follows in the same way as in Theorem 2.7. Fix an index $i_{0} \in I$ and a strong lifting $\rho_{i_{0}} \in \Lambda\left(\widehat{v}_{i_{0}}\right)$, where $\widehat{v}_{i_{0}}:=\widehat{v}_{\left\{i_{0}\right\}}$. Assume that $I=$ $\kappa:=\kappa(I)$ and $i_{0}=0$. Then $\left\langle X_{<\alpha}, \mathcal{S}_{<\alpha}, \Xi_{<\alpha}, v_{\alpha}, f_{(<\alpha)(<\beta)}, \kappa\right\rangle$ is a continuous projective system of topological probability spaces, $\left(X_{I}, \mathcal{S}_{I}, \Xi_{I}, v,\left\langle f_{<\alpha}\right\rangle_{\alpha<\kappa}\right)$ is its projective limit and the existence of a strong $\rho \in \Lambda(\widehat{\mu})$ follows exactly as in Theorem 4.5.

The assertion $(a)$ follows in the same way as in the proof of Theorem 2.7.

(b) Since $\Xi_{K}=\mathcal{B}_{0}\left(X_{K}\right)$ for every finite $K \subseteq I$, it follows by Lemma 5.1 that $\Xi_{J}=\mathcal{B}_{0}\left(X_{J}\right)$ for every $J \subseteq I$. On the other hand, the completion regularity of $v_{J}$ for every $J \in[I]^{\kappa}$ and assertion $(a)$ imply that $\widehat{\Xi}_{I}=\widehat{\mathcal{B}}_{0}\left(X_{I}\right)=\widehat{\mathcal{B}}\left(X_{I}\right)$ hence $v$ is completion regular and $\rho$ is completion Baire.

It remains an open question if assertion $(b)$ in the above corollary remains true under the assumption " $v_{K}$ is completion regular for every finite $K \subseteq I$ " instead of " $v_{J}$ is completion regular for every $J \in[I]^{<\kappa " \text {. }}$

THEOREM 5.3. Fix an index $i_{0} \in I$ and assume that the inclusion $\mathcal{S}_{i_{0}} \subseteq \widehat{\mathbb{\Xi}}_{i_{0}}$ holds true, the measure $v_{i_{0}}:=v_{\left\{i_{0}\right\}}$ admits a strong lifting $\rho_{i_{0}}$, and that for each $i \in I \backslash\left\{i_{0}\right\}$ the topological space $\left(X_{i}, \mathcal{S}_{i}\right)$ has a countable network consisting of sets in $\widehat{\Xi}_{i}$ and the measure $v_{i}$ supports $X_{i}$. Assume also that for every $J \in[I]^{<\kappa}$ we have $\mathcal{S}_{J} \subseteq \widehat{\Xi}_{J}$. If $v$ is a probability measure on $\Xi_{I}$, then the following conditions are equivalent:

(i) Condition (IT) for $\widehat{v}$.

(ii) Condition $(G I T)$ for $\widehat{v}$.

Each of them yields

(ii) There exists a strong lifting $\rho \in \Lambda(\widehat{v})$.

If (iii) is satisfied then:

(a) $\widehat{\Xi}_{J}=\widehat{\mathcal{B}}\left(X_{J}\right)$ for every $J \in[I]^{<\kappa}$ implies $\widehat{\Xi}_{I}=\widehat{\mathcal{B}}\left(X_{I}\right)$;

(b) If $\Xi_{i}=\mathcal{B}_{0}\left(X_{i}\right)$ for every $i \in I$ and $\left(X_{i}, \mathcal{S}_{i}\right)$ has a countable network consisting of sets in $\mathcal{B}\left(X_{i}\right)$, then $v_{i}$ is completion regular for every $i \in I, \Xi_{J}=\mathcal{B}_{0}\left(X_{J}\right)$ for every $J \subseteq I$ and $v$ is completion regular (hence $\rho$ is completion Baire).

Proof. If $I$ is finite then the result follows by Theorem 2.7 and a finite induction. So we may assume without loss of generality that $I$ is infinite. Moreover we may assume that $I=\kappa:=\kappa(I)$ and $i_{0}=0$. Then $\left\langle X_{<\alpha}, \mathcal{S}_{<\alpha}, \Xi_{<\alpha}, v_{\alpha}, f_{(<\alpha)(<\beta)}, \kappa\right\rangle$ is a continuous projective system of topological probability spaces and $\left(X_{I}, \mathcal{S}_{I}, \Xi_{I}, v,\left\langle f_{<\alpha}\right\rangle_{\alpha<\kappa}\right)$ is its projective limit.

The equivalence (i) $\Longleftrightarrow$ (ii) follows in the same way as in the proof of Theorem 5.2. It remains to show that (ii) $\Longrightarrow$ (iii). To this aim we construct by induction on $\delta<\kappa$ a consistent family $\left\langle\rho_{\delta}\right\rangle_{\delta<\kappa}$ of strong liftings $\rho_{\delta} \in \Lambda\left(\widehat{v}_{\delta}\right)$. The induction starts with $\rho_{i_{0}} \in \Lambda\left(\widehat{v}_{i_{0}}\right)$.

(A) Inductive step to a limit ordinal ordinal $\delta$. It follows by Proposition 4.2 that there exists a strong lifting $\rho_{\delta} \in \Lambda\left(\widehat{v}_{\delta}\right) \cap \operatorname{proj}_{\alpha<\delta} \lim \rho_{\alpha}$.

(B) Inductive step to a successor ordinal $\delta=\alpha+1<\kappa(\alpha \geq 0)$. Assume that $\rho_{\alpha} \in$ $\Lambda\left(\widehat{v}_{\alpha}\right)$ has been constructed. It then follows by Theorem 2.7 that there exists a strong inverse image $\rho_{\delta} \in \Lambda\left(\widehat{v}_{\delta}\right)$ of $\rho_{\alpha}$ under $f_{\alpha \delta}$.

(C) Since $\left\langle\rho_{\delta}\right\rangle_{\delta<\kappa}$ is a consistent family of strong liftings, it follows by Proposition 4.2 that there exists a strong lifting $\rho \in \Lambda(\widehat{v}) \cap \operatorname{proj} \delta_{\delta<} \lim \rho_{\delta}$. This completes the proof of the implication (ii) $\Longrightarrow$ (iii).

If the condition (iii) holds true, then the assertion (a) follows in the same way as in the proof of Theorem 5.2. 
If $\Xi_{i}=\mathcal{B}_{0}\left(X_{i}\right)$ for every $i \in I$ then this fact combined with the assumption $\mathcal{S}_{i} \subseteq \widehat{\Xi}_{i}$ implies that $v_{i}$ is completion regular for every $i \in I$. From Lemma 2.6 and a finite induction we get that $\mathcal{B}_{0}\left(X_{F}\right)=\bigotimes_{i \in F} \mathcal{B}_{0}\left(X_{i}\right)$ for each finite subset $F$ of $I$. So we may apply Lemma 5.1 to get $\mathcal{B}_{0}\left(X_{J}\right)=\Xi_{J}=\bigotimes_{i \in J} \mathcal{B}_{0}\left(X_{i}\right)$ for each $J \subseteq I$, hence $\widehat{\mathcal{B}}_{0}\left(X_{I}\right)=\widehat{\Xi}_{I}$, which combined with $(a)$ implies that $\widehat{\mathcal{B}}_{0}\left(X_{I}\right)=\widehat{\mathcal{B}}\left(X_{I}\right)$ and therefore $v$ is completion regular and $\rho$ is completion Baire.

The following immediate consequence of Theorem 5.3 is a result of A. and C. Ionescu Tulcea [14, Chapter VIII, Section 2, Theorem 5 and its corollary].

COROLlary 5.4. Let $\left\langle X_{i}\right\rangle_{i \in I}$ be a family of compact topological spaces and let $v$ be a positive Radon measure on $X_{I}$ supporting $X_{I}$. Suppose that

(i) $X_{i}$ is metrizable for each $i \in I$,

(ii) v satisfies condition (IT).

Then $v$ has a strong lifting.

The next immediate consequence of Theorem 5.3 is a result of Fremlin [9, Proposition 453I].

COROLlary 5.5. Let $\left\langle\left(X_{i}, \mathcal{S}_{i}, \Xi_{i}, v_{i}\right)\right\rangle_{i \in I}$ be a family of complete topological probability spaces such that every $\left(X_{i}, \mathcal{S}_{i}\right)$ has a countable network consisting of measurable sets and every $v_{i}$ has full support. Let $v_{I}$ be the product measure on $X_{I}$. Then $\widehat{v}_{I}$ is a $\tau$-additive measure and has a strong lifting.

COROLlaRY 5.6. Let $(\Omega, \mathcal{T}, \Sigma, \mu)$ be a topological probability space such that $\widehat{\mu}$ admits a strong lifting $\rho$, and assume that each $\left(X_{i}, \mathcal{S}_{i}\right)$ has a countable network consisting of sets in $\widehat{\Xi}_{i}$ and each $\nu_{i}$ supports $X_{i}$. Assume also that $\mu \widehat{\otimes} \nu$, as a measure on the product of the $\sigma$-algebras $\Sigma$ and all $\Xi_{i}, i \in I$, satisfies condition $(I T)$. Then $\mu \widehat{\otimes} v$ admits $a$ strong lifting $\varphi$ being an inverse image of $\rho$ under the canonical projection from $\Omega \times X_{I}$ onto $\Omega$.

In particular, if $\Sigma=\mathcal{B}_{0}(\Omega)$, for each $i \in I$ the equality $\Xi_{i}=\mathcal{B}_{0}\left(X_{i}\right)$ holds true and $\left(X_{i}, \mathcal{S}_{i}\right)$ has a countable network consisting of sets from $\mathcal{B}\left(X_{i}\right)$, then $\Sigma \otimes \Xi_{I}=$ $\mathcal{B}_{0}\left(\Omega \times X_{I}\right), \mu \otimes v$ is completion regular and $\varphi$ is in addition completion Baire.

Proof. Let $J$ be an arbitrary index set, $i_{0} \in J \backslash I$ and $\left(X_{i_{0}}, \mathcal{S}_{i_{0}}, \Xi_{i_{0}}, v_{i_{0}}\right)=$ $(\Omega, \mathcal{T}, \Sigma, \mu)$. Put $I_{0}:=I \cup\left\{i_{0}\right\}$. Then the families $\left\langle\left(X_{i}, \mathcal{S}\right)\right\rangle_{i \in I_{0}}$ and $\left\langle\Xi_{i}\right\rangle_{i \in I_{0}}$ together with the probability measure $\mu \otimes v$ on $\Xi_{I}$ satisfy the assumptions of Theorem 5.3 and so the result follows.

The above corollary contains Theorem 3 of [4].

THEOREM 5.7. Fix an index $i_{0} \in I$ and assume that the measure $v_{i_{0}}$ admits a strong lifting $\rho_{i_{0}}$. Assume that for every $i \in \Lambda\left\{i_{0}\right\}$ there exists a family $\left\langle\left(X_{j}^{i}, \mathcal{S}_{j}^{i}\right)\right\rangle_{j \in I_{i}}$ of topological spaces and a family $\left\langle\left(\Xi_{j}^{i}\right\rangle_{j \in I_{i}}\right.$ of $\sigma$-algebras $\Xi_{J}^{i}$ on $X_{j}^{i}$, respectively, such that for every $j \in I_{i}$ the space $\left(X_{j}^{i}, \mathcal{S}_{j}^{i}\right)$ has a countable network consisting of sets in $\widehat{\Xi}_{j}^{i}$. Assume that $X_{i}=\otimes_{j \in I_{i}} X_{j}^{i}, \mathcal{S}_{i}=\otimes_{j \in I_{i}} \mathcal{S}_{j}^{i}$ and $\Xi_{i}=\otimes_{j \in I_{i}} \Xi_{j}^{i}$. Assume also that for every $J \in[I]^{<\kappa}$ and for every $i \in I \backslash J$ the measure $\widehat{v}_{J \cup\{i\}}$, as a measure on the completed product of the $\sigma$ algebras $\Xi_{J}$ and $\Xi_{j}^{i}$ for all $j \in I_{i}$, satisfies condition $(I T)$. Then the measure $\widehat{v}$ admits a strong lifting.

Proof. If $I$ is finite then the result follows from Theorem 5.3 and finite induction. So we may assume that $I$ is infinite. We may also assume that $I=\kappa:=\kappa(I)$ and $i_{0}=0$. 
Then $\left\langle X_{<\alpha}, \mathcal{S}_{<\alpha}, \Xi_{<\alpha}, v_{\alpha}, f_{(<\alpha)(<\beta)}, \kappa\right\rangle$ is a continuous projective system of topological probability spaces and $\left(X_{I}, \mathcal{S}_{I}, \Xi_{I}, v,\left\langle f_{<\alpha}\right\rangle_{\alpha<\kappa}\right)$ is its projective limit.

We construct by induction on $\delta<\kappa$ a consistent family $\left\langle\rho_{\delta}\right\rangle_{\delta<\kappa}$ of strong liftings $\rho_{\delta} \in \Lambda\left(\widehat{v}_{\delta}\right)$. The induction starts with $\rho_{i_{0}} \in \Lambda\left(\widehat{v}_{i_{0}}\right)$. The rest of the proof follows in the same way as in Theorem 5.3, proof of the implication $($ ii $) \Longrightarrow$ (iii). We have only to replace Theorem 2.7 by Theorem 5.3.

The first result on completion regularity is the following classical theorem of Kakutani [15]: If $\left\langle X_{i}\right\rangle_{i \in I}$ is a family of compact metric spaces and for every $i \in I, v_{i}$ is a Radon probability measure on $X_{i}$ with full support, then the Radon product measure of $\left\langle v_{i}\right\rangle_{i \in I}$ on $X_{I}$ is completion regular. An immediate consequence of Theorem 5.7 is the following generalization of the above classical result of [15].

COROLlary 5.8. Under the assumptions of Theorem 5.7 and the assumptions that $\Xi_{i_{0}}=\mathcal{B}_{0}\left(X_{i_{0}}\right)$, for every $i \in I \backslash\left\{i_{0}\right\}$ the equality $\Xi_{j}^{i}=\mathcal{B}_{0}\left(X_{j}^{i}\right)$ holds true and $\left(X_{j}^{i}, \mathcal{S}_{j}^{i}\right)$ has a countable network consisting of sets from $\mathcal{B}\left(X_{j}^{i}\right)$, it follows that for every $J \subseteq I$ the equality $\Xi_{J}=\mathcal{B}_{0}\left(X_{J}\right)$ holds true, the measure $v$ is completion regular and there exists a strong completion Baire lifting for $\widehat{v}$.

Proof. According to Theorem 5.3 we have that for each $i \in I$ the equality $\Xi_{i}=$ $\mathcal{B}_{0}\left(X_{i}\right)$ holds true, the measure $v_{i}$ is completion regular and $\widehat{v}_{i}$ admits a strong lifting. Again by Theorem 5.3 and finite induction we get that $\mathcal{B}_{0}\left(X_{F}\right)=\bigotimes_{i \in F} \mathcal{B}_{0}\left(X_{i}\right)$ for every finite $F \subseteq I$. On the other hand, according to Theorem 5.7 the measure $\widehat{v}$ admits a strong p-lifting $\rho$, it is $\tau$-additive, and the inclusion $\mathcal{S}_{I} \subseteq \widehat{\Xi}_{I}$ holds true. So we may apply Lemma 5.1 to get the equality $\mathcal{B}_{0}\left(X_{J}\right)=\otimes_{i \in J} \mathcal{B}_{0}\left(X_{i}\right)$ for each $J \subseteq I$, hence $\Xi_{I}=$ $\otimes_{i \in I} \mathcal{B}_{0}\left(X_{i}\right)=\mathcal{B}_{0}\left(X_{I}\right)$. Consequently, $\widehat{\Xi}_{I}=\widehat{\mathcal{B}}_{0}\left(X_{I}\right)$, which combined with the inclusion $\mathcal{S}_{I} \subseteq \widehat{\Xi}_{I}$ implies $\widehat{\mathcal{B}}\left(X_{I}\right) \subseteq \widehat{\mathcal{B}}_{0}\left(X_{I}\right)$ hence $\widehat{\mathcal{B}}\left(X_{I}\right)=\widehat{\mathcal{B}}_{0}\left(X_{I}\right)$. Thus, $v$ is completion regular and $\rho$ is in addition completion Baire.

The following result, which is a consequence of Theorem 5.7 and of [12, Theorem 3.1], is related with Question 5.5.

COROLlary 5.9. Let $(\Omega, \mathcal{T}, \widehat{\mathcal{B}}(\Omega), \mu)$ be a complete topological probability space such that $(\Omega, \mathcal{T})$ is completely regular and $\mu$ is completion regular and $\tau$-additive. Assume that for every $i \in I$ there exists a family $\left\langle\left(X_{j}^{i}, \mathcal{S}_{j}^{i}\right)\right\rangle_{j \in I_{i}}$ of separable metric spaces and $v_{i}$ has full support, and that for every $J \in[I]^{<\kappa}$ and for every $i \in I \backslash J$ the measure $\widehat{v}_{J \cup\{i\}}$, as a measure on the completed product of $\Xi_{J}$ and $\mathcal{B}\left(X_{j}^{i}\right)$ for all $j \in I_{i}$, satisfies condition (IT). Then the $\tau$-additive product measure (see [12, page 331] for the definition) of $\mu$ and $\otimes_{i \in I} v_{i}$ is completion regular.

Proof. By Corollary 5.8 it follows that $v_{I}$ is completion regular. So the result follows from [12, Theorem 3.1].

REMARKS 5.10. (a) It should be noted that according to [14, page 120], a measure $\mu$ satisfying the property $(I T)$ as in Theorem 5.2 is not in general equivalent with a product measure.

(b) The measure $\mu$ of Example 4.3 does not satisfy condition (IT) of Theorems 5.2 and of 5.3 .

In fact, assuming that $\mu$ satisfies condition $(I T)$ and that the continuum hypothesis $(\mathrm{CH})$ is satisfied, we get from the above corollary the completion regularity of $\mu$, a contradiction. 
It should be noted that subject to $(\mathrm{CH})$ the measure $\mu$ admits a strong lifting (see [7] or [28]).

(c) Subject to $(\mathrm{CH})$, the Wiener measure $W$ defined on the completion of the Borel $\sigma$-algebra of $\Omega:=\overline{\mathbf{R}}_{\alpha}^{[0,1]}=\overline{\mathbf{R}}^{\omega_{1}}$ where $\overline{\mathbf{R}}=[+\infty,-\infty]$ does not satisfy condition $(I T)$. In fact, let $\Omega_{\alpha}:=\overline{\mathbf{R}}^{\alpha}, f_{\alpha}$ be the canonical projection of $\overline{\mathbf{R}}^{\omega_{1}}$ onto $\overline{\mathbf{R}}^{\alpha}$ for every $\alpha<\omega_{1}$ and, $W_{\alpha}$ be the completed image of $W$ under $f_{\alpha}$.

Since each $W_{\alpha}$ has the $U S L P$, supports $\Omega_{\alpha}$, and is completion regular but $W$ is not completion regular, it follows by Corollary 5.6 that $\mu$ does not satisfy condition (IT). The measure $W$ has the USLP because the support of $W$ is the polish space $C(\overline{\mathbf{R}}) . \Omega$ is not second countable, because if $\Omega$ were second countable then $W$ should be completion regular, a contradiction. Moreover, $\Omega$ has no countable network (see [13] for the definition) since for compact spaces $\Omega$ the net weight, i.e. the least cardinality of a network for $\Omega$, is equal to the weight of $\Omega$ (see Gruenhage [13, Chapter 10, Section 1)]. So $(X, \widehat{\mathcal{B}}(\Omega), W)$ is an example of a topological probability space with the USLP but without countable base and without countable network.

(d) Fremlin's simplification [8] of Losert's counter-example to the strong lifting conjecture gives a Radon probability measure $\mu$ on $\Omega:=\{0,1\}^{\aleph_{2}}$ supporting $\Omega$, being completion regular and having no a strong lifting. It follows by Theorem 5.3 and above that the measure $\mu$ does not satisfy condition $(I T)$.

6. Consequences. We recall the following definition taken from [32, Chapter I, Section 6, page 46]. Let $v$ be a Radon measure on a topological space $(\Theta, \mathcal{S})$. A $v$ concassage of $\Theta$ is a partition of $\Theta$ into $N \cup \bigcup_{i \in I} K_{i}$, where $N$ is a $\nu$-null set and $K_{i}$ are pairwise disjoint compact sets such that every point of $\Theta$ has a neighbourhood which meets at most a countable number of $K_{i}$.

Let $\kappa$ be an infinite ordinal. We say that $(\Theta, \nu)$ is locally of order $\kappa$, if there exists a concassage $\left\langle\left\langle K_{i}\right\rangle_{i \in I}, N\right\rangle$ of $\Theta$ such that the topology induced on every $K_{i}$ has a basis of cardinality not exceeding $\kappa$ (see [2]).

The following result has been proved in [2, page 22] for Radon measures of full support on a locally compact Hausdorff topological space, but we extend it to general (Hausdorff) topological spaces. Although the proof essentially repeats the arguments of [2] we give it for completeness.

LEMMA 6.1. Let $\kappa$ be an infinite ordinal. In order that every (completion regular and) Radon measure $v$ of full support on a (normal) topological space $\Theta$, such that $(\Theta, v)$ is locally of order $\kappa$, has a strong (completion Baire) lifting it is necessary and sufficient that every (completion regular and) Radon measure $v$ of full support on $[0,1]^{\kappa}$ has a strong (completion Baire) lifting.

Proof. The necessity is obvious. Let $v$ be a Radon measure of full support on $\Theta$. Suppose that $(\Theta, v)$ is locally of order $\kappa$. Applying [32, Chapter I, Section 6, Theorem 13], we may choose a concassage $\left\langle\left\langle K_{i}\right\rangle_{i \in I}, N\right\rangle$ of $\Theta$ such that the restriction $v \mid K_{i}$ of $v$ to each $K_{i}$ is of full support. Indeed, let $\left\langle\left\langle L_{i}\right\rangle_{i \in I}, N\right\rangle$ be a concassage of $\Theta$. For every $i \in I$ let $K_{i} \subseteq L_{i}$ be the support of $\nu \mid L_{i}$. Since each $L_{i}$ has a basis of cardinality less or equal to $\kappa$, the same holds true for $K_{i}$. Exactly as in Schwartz [32] one proves that $\Theta \backslash \bigcup_{i} K_{i}$ is $\nu$-measurable. We want to prove that $v\left(\Theta \backslash \bigcup_{i} K_{i}\right)=0$. So take a compact $H \subset \Theta \backslash \bigcup_{i} K_{i}$ and take for every $\theta \in H$ a neighborhood $U_{\theta}$ such that $U_{\theta}$ has non-empty intersection only with countably many sets $L_{\theta, i}$. Due to the compactness of $H$ there exist sets $U_{1}, \ldots, U_{n} \in\left\{L_{\theta, i}: \theta \in \Theta, i \in I\right\}$ covering $H$. Assume that 
$U_{m} \cap L_{m, j} \neq \emptyset, j=1, \ldots$ and $U_{m} \cap L_{j}=\emptyset$ for other $L_{j}$. Thus, $U_{m} \cap\left(\Theta \backslash \bigcup_{i, j} L_{i, j} \backslash N\right)=$ $\emptyset$ for every $m \leq n$. Consequently, $H \cap\left(\Theta \backslash \bigcup_{i, j} L_{i, j} \backslash N\right)=\emptyset$, what yields $H \subset \bigcup_{i, j} L_{i, j} \cup$ $N$. As $v\left(H \cap L_{i, j}\right)=0$ for all $i, j$, we get $v(H)=0$.

For each $i \in I$ there is a homeomorphism $h_{i}$ from $K_{i}$ onto a closed subset $h_{i}\left(K_{i}\right)$ of $[0,1]^{\kappa}$. Denote by $\widetilde{v}_{i}$ the image measure of $v \mid K_{i}$ under $h_{i}$ for $i \in I$, and by $\lambda$ the Lebesgue measure on $[0,1]$. Then $v_{i}:=\lambda^{\kappa}+\widetilde{v}_{i}$ supports $[0,1]^{\kappa}$ and by our assumption there exists a strong lifting $\bar{\rho}_{i} \in \Lambda\left(v_{i}\right)$.

Since $v \mid K_{i}$ supports $K_{i}$, it follows that $\widetilde{v}_{i} \mid h_{i}\left(K_{i}\right)$ supports $h_{i}\left(K_{i}\right)$ for each $i \in I$, hence we may apply $\left[17\right.$, Lemma 2.3], to find a strong lifting in $\Lambda\left(v_{i} \mid \widehat{\mathcal{B}}\left([0,1]^{\kappa}\right) \cap h_{i}\left(K_{i}\right)\right)$, and again [17, Corollary 2.6], to find a strong lifting $\rho_{i} \in \Lambda\left(\widetilde{v}_{i} \mid h_{i}\left(K_{i}\right)\right)$. Since $h_{i}$ is a homeomorhism there exists an inverse image $\tau_{i} \in \Lambda\left(v \mid K_{i}\right)$ of $\rho_{i}$ under $h_{i}$ for each $i \in I$, which is a strong lifting. It then follows from [17, Theorem 2.4], that there exists a strong lifting in $\Lambda(v)$.

If in addition $\Theta$ is a normal topological space and $v$ is completion regular then it follows from [6, Lemma 2.3] that for each $i \in I$ we have $\mathcal{B}_{0}\left(K_{i}\right)=\mathcal{B}_{0} \cap K_{i}$ according to [6, Lemma 2.3], hence the measure $v \mid K_{i}$ is completion regular, and so applying [4, Section 3, Lemma 1], we have that $v_{i}$ is completion regular, and again by [6, Lemma 2.3], $v_{i} \mid h_{i}\left(K_{i}\right)$ is completion regular. Consequently, the above liftings $\bar{\rho}_{i}, \widetilde{\rho}_{i}, \rho_{i}, \tau_{i}$ and $\tau$ are strong completion Baire and so the result follows.

QUESTION 6.2. Can we eliminate the completeness assumption for the topological measure spaces in Lemma 6.1?

DEFINITION 6.3. Let $\kappa$ be an infinite ordinal and let $v$ be a Radon measure on a topological space $(\Theta, \mathcal{S})$ such that $(\Theta, v)$ is locally of order $\kappa$. Let $\left\langle v_{i}\right\rangle_{i \in I}$ be the family of the measures $v_{i}$ on $[0,1]^{\kappa}$ associated with $(\Theta, v)$ as in the proof of Lemma 6.1. We say that the measure $v$ satisfies the property $\left(G P^{*}\right)$, if for every $i \in I$ the measure $v_{i}$ satisfies the property $(I T)$.

THEOREM 6.4. Let $v$ be an arbitrary (completion regular and) Radon measure of full support on a (normal) toplogical space $\Theta$ satisfying the property $\left(G P^{*}\right)$. Then $v$ admits a strong (completion Baire) lifting.

Proof. Let $(\Theta, v)$ be of locally order $\kappa$, where $\kappa$ is an infinite ordinal. Then the result follows by Lemma 6.1 and by Theorem 5.3.

THEOREM 6.5. Let $\mu$ and $v$ be Radon measures of full support on the topological spaces $(\Omega, \mathcal{T})$ and $(\Theta, \mathcal{S})$ respectively such that $(\Theta, \nu)$ is locally of order $\kappa$, where $\kappa$ is an arbitrary infinite ordinal. Let $\left\langle v_{i}\right\rangle_{i \in I}$ be the family of the measures $v_{i}$ on $[0,1]^{\kappa}$ associated with $(\Theta, v)$ as in the definition 6.2. Assume that $\mu$ admits a strong lifting $\rho$ and $\mu \widehat{\otimes} \nu$ has the property $\left(G P^{*}\right)$. Then $\mu \widehat{\otimes} v$ admits a strong lifting being an inverse image of $\rho$ under the canonical projection.

In particular, if $\mu$ and $v$ are completion regular and $(\Theta, \mathcal{S})$ is a normal topological space, then the measure $\mu \widehat{\otimes} v$ admits a strong completion Baire lifting being an inverse image of $\rho$ under the canonical projection.

Proof. Let $\kappa$ be an infinite ordinal such that $(\Theta, \mathcal{S})$ is locally of order $\kappa$. As in the proof of Lemma 6.1, there exists a concassage $\left\langle\left\langle K_{i}\right\rangle_{i \in I}, N\right\rangle$ of $\Theta$ such that the restriction $v \mid K_{i}$ of $v$ to each $K_{i}$ is of full support and each topology $\mathcal{S} \cap K_{i}$ has a basis not exceeding $\kappa$. For each $i \in I$ there is a homeomorhism $h_{i}$ from $K_{i}$ onto a closed subset $h_{i}\left(K_{i}\right)$ of $[0,1]^{\kappa}$. According to Corollary 5.6, for each $i \in I$ the measure 
$\mu \widehat{\otimes} v_{i}$ has a strong p-lifting, and by [17, Lemma 2.3], the restriction of $\mu \widehat{\otimes} v_{i}$ to $\widehat{\mathcal{B}}\left(\Omega \times h_{i}\left(K_{i}\right)\right)$, has a strong lifting. But again by [17, Corollary 2.6], the restriction of $\mu \widehat{\otimes} \widetilde{v}_{i}$ to $\widehat{\mathcal{B}}\left(\Omega \times h_{i}\left(K_{i}\right)\right)$ has a strong lifting, and so since $h_{i}$ is a homeomorphism we get that the measure $\mu \widehat{\otimes}\left(\nu \mid K_{i}\right)$ admits a strong lifting for each $i \in I$.

Consequently, applying [17, Theorem 2.4] we get a strong lifting for $\mu \widehat{\otimes} \nu$ being an inverse image of $\rho$.

In particular, if $\mu$ and $v$ are completion regular and $(\Theta, \mathcal{S})$ is a normal topological space, then according to Corollary 5.6, for each $i \in I$ the measure $\mu \widehat{\otimes} \nu_{i}$ has a strong completion Baire p-lifting, and by [17, Lemma 2.3], the restriction of $\mu \widehat{\otimes} v_{i}$ to $\widehat{\mathcal{B}}(\Omega \times$ $h_{i}\left(K_{i}\right)$ ), which according to [6, Lemma 2.3], is completion regular, has a strong lifting. But again by [17, Corollary 2.6], the restriction of $\mu \widehat{\otimes} \widetilde{v}_{i}$ to $\widehat{\mathcal{B}}\left(\Omega \times h_{i}\left(K_{i}\right)\right)$, which is completion regular, has a strong lifting, and so since $h_{i}$ is a homeomorphism we get that the measure $\mu \widehat{\otimes}\left(v \mid K_{i}\right)$ admits a strong completion Baire lifting for each $i \in I$.

Consequently, applying [17, Theorem 2.4] we get a strong completion Baire lifting for $\mu \widehat{\otimes} v$ being an inverse image of $\rho$. This completes the proof.

The above theorem extends Corollary 5.6, while Theorem 6.4 improves Theorem 5.3.

Theorem 6.5 gives a positive answer to Kupka's problem for a wide class of topological probability spaces. The same theorem answers Question 2.3 to the positive for Radon measure spaces $(\Theta, \mathcal{S}, \widehat{\mathcal{B}}(\Theta), v)$ and

$$
(\Omega, \mathcal{T}, \widehat{\mathcal{B}}(\Omega), \mu)=(\Theta, \mathcal{S}, \widehat{\mathcal{B}}(\Theta), \nu) \widehat{\otimes}(\widetilde{\Theta}, \widetilde{\mathcal{S}}, \widehat{\mathcal{B}}(\widetilde{\Theta}), \widetilde{v})
$$

where $(\widetilde{\Theta}, \widetilde{\mathcal{S}}, \widehat{\mathcal{B}}(\widetilde{\Theta}), \widetilde{v})$ is as in Theorem 6.4 .

\section{REFERENCES}

1. A. G. A. G. Babiker and W. Strauss, Almost strong liftings and $\tau$-additivity, in Measure Theory Proceedings Oberwolfach 1979 (Ed. D. Kölzow), Lecture Notes in Math. No 794 (Springer Verlag, 1980), 220-227.

2. K. Bichteler, An existence theorem for strong liftings, J. Math. Anal. Appl. 33 (1971), $20-22$.

3. J. R. Choksi, Inverse limits of measure spaces, Proc. London Math. Soc. (3) 8 (1958), 321-342.

4. J. R. Choksi and D. H. Fremlin, Completion regular measure on product spaces, Math. Ann 241 (1979), 113-128.

5. J. Dugundji, Topology (Allyn and Bacon, Inc., Boston, 1970).

6. G. D. Edgar, Measurable weak sections, Illinois J. Math. 20 (1976), 630-646.

7. D. H. Fremlin, On two Theorems of Mokobodzki, Note of 23 June 1977, University of Essex, Mathematics Department.

8. D. H. Fremlin, Losert's example, Note of 18/9/79, University of Essex, Mathematics Department.

9. D. H. Fremlin, Measure theory, to appear.

10. S. Graf and H. von Weizsäcker, On the existence of lower densities in non-complete measure spaces, in Measure Theory Proceedings Oberwolfach 1975 (ed. by A. Bellow and D. Kölzow), Lecture Notes in Math. No 541 (Springer Verlag, 1976), 155-158. 71-84.

11. C. Gryllakis and S. Grekas, On products of Radon measures, Fund. Math. 159 (1999),

12. C. Gryllakis and G. Koumoullis, Completion regularity and $\tau$-additivity of measures on product spaces, Compositio Math. 73 (1990), 329-344. 
13. G. Gruenhage, Generalized metric spaces, in Handbook of set-theoretic toplogy, editors K. Kunen and J. E. Vaughan (Elsivier Science Publishers., 1984).

14. A. and C. Ionescu Tulcea, Topics in the theory of lifting (Springer-Verlag, 1969).

15. S. Kakutani, Notes on infinite product measure spaces II, Proc. Imperial Acad. Tokyo 19 (1943), 184-188.

16. H. G. Kellerer, Baire sets in product spaces, in Measure Theory Proceedings Oberwolfach 1979 (ed. by D. Kölzow), Lecture Notes in Math. No. 794 (Springer-Verlag, 1980), 38-44.

17. J. Kupka, Strong liftings with applications to measurable cross sections in locally compact groups, Israel J. Math. 44 (1983), 243-261.

18. N. D. Macheras, K. Musiał and W. Strauss, On products of admissible liftings and densities, Z. Anal. Anwendungen 18 (1999), 651-667

19. N. D. Macheras, K. Musiał and W. Strauss, Linear liftings respecting coordinates, $A d v$. Math. 153 (2000), 403-416.

20. N. D. Macheras, K. Musiał and W. Strauss, Strong admissibly generated liftings and products of liftings, Atti. Sem. Mat. Fis. Univ. Modena 50 (2002), 217-224.

21. N. D. Macheras and W. Strauss, On various strong lifting properties for topological measure spaces, Rend. Circolo Mat. Palermo (2) Suppl. 28 (1992), 149-162.

22. N. D. Macheras and W. Strauss, On strong liftings for projective limits, Fund. Math. 144 (1994), 209-229.

23. N. D. Macheras and W. Strauss, On products of almost strong liftings, J. Austral. Math. Soc. Ser. A 60 (1996), 1-23.

24. N. D. Macheras and W. Strauss, Products of lower densities, Z. Anal. Anwendungen. 14 (1995), 25-32.

25. N. D. Macheras and W. Strauss, Products and projective limits of almost strong liftings, Atti. Sem. Mat. Fis. Univ. Modena 44 (1996), 119-133.

26. N. D. Macheras and W. Strauss, On consistent families of almost strong liftings in projective systems, Atti Sem. Mat. Fis. Univ. Modena 44 (1996), 105-111.

27. N. D. Macheras and W. Strauss, On strong product liftings, Atti Sem. Mat. Fis. Univ. Modena 50 (2002), 37-52.

28. G. Mokobodzki, Relèvement borelien compatible avec une classe d'ensembles négligeables. Application à la désintegration des mesures, Seminaire des probabilites IX, 1974/75, Lecture Notes in Math. No. 465 (Springer-Verlag, 1975), 437-442.

29. K. Musiał, Projective limits of perfect measure spaces, Fund. Math. 110 (1980), 163-189.

30. J. von Neumann, Algebraische Repräsentanten der Funktionen bis auf eine Menge von Masse Null, J. Reine Angew. Math. 165 (1931), 109-115.

31. K. H. Ross and A. H. Stone, Products of separable spaces, Amer. Math. Monthly 71 (1964), 398-403.

32. L. Schwartz, Radon measures on arbitrary topological spaces and cylindrical measures (Oxford University Press, 1973). 